S B

$\mathrm{M} 2 \mathrm{C}$ 


$$
\text { \% }
$$







\title{
Itate of Connecticut
}

REPORT

OF

The Connecticut Agricultural

Experiment Station

NEW HAVEN, CONN.

\section{INHERITANCE IN CORN}

BEING PART VI OF THE ANNUAL REPORT OF 1911

\author{
JUNE, 1912 \\ CONCLUSION
}

Seprovate

Thograph 



\title{
PART VI.
}

\section{INHERITANCE IN CORN.}

\author{
By H. K. HAYES.
}

\section{Introduction}

For the last six years this Station has been studying the inheritance of corn characters, and in IgII a technical bulletin was published on the subject. The purpose of this paper is to state as clearly as possible some of our results which have practical value to corn growers and breeders in two different particulars.

First, a large number of corn varieties are grown in Connecticut and often several of them on the same farm. In spite of usual precautions, slight accidental crossings between varieties take place which cause serious embarrassment to growers of seed corn and plague everyone who wants to raise corn which is uniform in appearance. In the following pages is stated what can be done to detect and cull out the accidental hybrid seed.

Second, many more or less successful attempts have been and are being made to develop new hybrid varieties which shall combine desirable qualities from both parents. Accurate knowledge of the way in which these desirable qualities are inherited will simplify operations and prevent disappointment.

\section{The Formation of the Seed}

Following the general rule, seed corn can only be produced by a union of male and female elements. The tassels and silks of corn, which are the male and female reproductive organs, are borne on widely separated parts of the plant. Each thread of silk grows from a spot on the cob where the kernel is to be and where the female cells are produced. The office of the silk is to collect the pollen grains which are formed in the tassel. A mature pollen grain falling on a mature silk germinates and sends out a tube which, guided by the silk, reaches the ovary. Through this tube two male cells pass, and on reaching the female cells, 
unite with them. One unites with the egg cell to form the embryo or undeveloped plant and the other fuses with a second female cell to form the endosperm of the seed. This endosperm is the surrounding tissue in which the food material is stored. The embryo of the corn seed is the germ or chit, the rest of the seed within the outer covering is the endosperm.

The fusion of the cells just described, called fertilization, is at once followed by rapid growth and the production of a seed. When the pollen comes from the same plant which bears the silk, there is "self-fertilization ;" when it comes from another plant of the same variety there is "cross-fertilization;" when from another variety there results "hybridization." As a commercial variety of corn is generally composed of many types in a complex hybrid condition, due to constant inter-crossing, there is no exact distinction between "cross-fertilization" and "hybridization" as applied to corn.

\section{The Law of Heredity}

The transmission from one generation to another of the prominent features, such as sweet, dent and flint characters, color of seeds, etc., which distinguish varieties of corn, follows what is known as Mendel's Law of Heredity, which may be illustrated from his own work with peas. For this discussion Darbishire's book, "Breeding and the Mendelian Discovery," has been freely used.

The edible pea may be divided into two classes, the tall and the dwarf. One difference between them is in the length of the internodes, i. e., the sections of the stem between two nodes or joints where the leaves are attached. This causes the tallness or the shortness of the whole plant.

Moreover, the pea is a normally self-fertilized plant, i. e., seed is formed by the union of male and female cells of the same plant.

This seed, if from a tall race, will produce nothing but tall plants, and if from a dwarf plant will yield dwarfs only.

Now, if a cross is made between a tall and a dwarf race by applying the pollen of one variety to the stigma or female receptive organ of another variety, and the seeds produced by this "cross" are sown, nothing but tall plants will appear. These are no shorter than the pure tall plants and in many cases they are 
somewhat taller because of the increased vigor due to crossing two pure races:

When we sow the seed from the above hybrid generation we obtain tall and dwarf plants in the ratio of about three tall to one dwarf in every four. These dwarfs, if self-fertilized, will all breed true as to height in later generations, but while some of the tall plants will breed true others will again give tall and dwarf plants in the ratio of three to one.

These facts are easily explained by the present Mendelian theory, which is not essentially different from Mendel's interpretation. A plant or animal does not transmit its characters in a bunch as it were, as if the entire organism were the unit, but its various characters are inherited separately.

Each reproductive cell, whether in pollen or ovule of the tall pea, contains a factor, structure or unit quality of tallness which we may represent by $T$. A union of male and female cells of tall peas will be a union of reproductive cells, all of which contain $T$, and a tall race will result. Each such reproductive cell of the dwarf pea lacks the structure or factor which produces the tall race. This lack may be represented by t, and from their fusion only dwarfs result.

But when the tall and dwarf races are crossed, each seed is the result of a union of reproductive cells, the one from the tall race containing $T$ and the one from the dwarf race containing $t$. But the tall character in this generation is "dominant;" $i$. e., it conceals or masks the other. Such characters as tallness and dwarfness are said to be contrasted or allelomorphic pairs, and as only tall plants are produced when tall and dwarf are crossed, the tall character is said to be a dominant one and the divarf character a recessive condition.

Now, if all the seeds of this hybrid are planted and their blossoms self-fertilized, we may explain the conditions as follows: Half of the male cells are supposed to contain only $T$, the factor for tallness, and half only $t$, the factor for dwarfness. The same is true of the female cells. We may represent the situation and the resulting progeny thus:

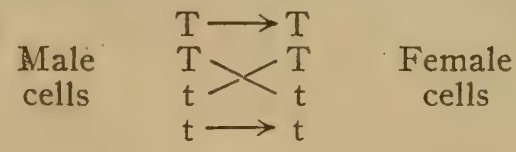


From the diagram it appears that one-quarter of the resulting seeds have received $T$ from both pollen and ovule, or $(T+T)$. They can produce only tall progeny. One-quarter have received $t$ from both, or $(t+t)$ and can produce only dwarf progeny. The remaining half of the seeds are like those from which they themselves were grown, i. e., they are formed by a union of cells, one of which contains $T$ and the other $t$, or $(T+t)$. But when the dominant factor $T$ is received from only one reproductive cell the plant when grown is a tall one and can not be distinguished from the pure tall race.

The only method of distinguishing between the pure tall and hybrid tall plants is by breeding from them. The pure tall plants will, of course, give only tall progeny, while the hybrid tall plants will again give both tall and dwarf plants.

The first important fact which this illustrates is that the external appearance of a plant is not a correct criterion of its breeding qualities, but that the contents of the reproductive cells are the important feature, and that in most cases the only way of determining these contents is to breed from them.

We should also note that only one of the factors of a contrasted pair is found in a single reproductive cell. Thus, in the case above described no reproductive cell consisted of a mixture of the factors for both tall and dwarf plants but contained either the one or the other.

The fact that there is often a dominance of one condition over another in the first hybrid generation of a cross is of especial importance to the corn growers of Connecticut. A partial report of this matter was given in 1907 by East.

\section{Dominance of Characters in Corn Breeding}

After crossing, the characters here given behave in the following manner:

Flint or Dent is dominant to Sweet.

Yellow

Purple

Red Pericarp

Red Cob

$\begin{array}{ll}66 & 66 \\ 66 & 66 \\ 66 & 66 \\ 66 & 66\end{array}$

"No Yellow or White.

" No Purple or White.

" No Red or White.

"No Red or White. 


\section{Flint or Dent-Sweet Crosses}

If a sweet corn is used as the female parent and is crossed with a starchy corn, either a flint or a dent, there result hybrid seeds which cannot be distinguished from seeds of the starchy parent. This result is due to the fact that the endosperm of corn is hybridized as well as the embryo. (See The Formation of the Seed.)

A microscopic study of the starch grains of sweet corn shows them to be small, angular and abortive, while those of a flint or dent are much larger and are circular in outline. Something is evidently introduced by the pollen of the starchy corn which enables the sweet variety to proceed in its starch development and form seeds which are not visibly different from the starchy parent.

If, on the other hand, starchy corn, flint or dent, is used as the female parent and is pollinated with sweet corn pollen, no immediate effect is apparent. The starchy corn already contains the dominant factor and masks or hides the presence of the sweet character which is recessive.

The practical use of these facts is as follows:

No extreme care need be used in isolating sweet corn plots from field corn, as any crossing on the sweet corn shows in the first year, and at harvest the hard starchy kernels can be discarded. The sweet kernels, those with a wrinkled appearance, when grown will always produce sweet corn.

But, on the other hand, as there is no immediate visible change when field corn is pollinated with sweet corn pollen, there is no method of detecting and rejecting the hybrid kernels. The field corn seed should always therefore be saved from that part of the field which is farthest from the sweet corn plot.

Sometimes seeds of a semi-starchy nature appear in sweet corn varieties. In a study of the inheritance of starchy-sweet crosses a few ears of this nature were met with. Semi-starchy seeds have also been mentioned as occurring in a number of pure sweet races by Halsted of New Jersey. To determine the inheritance of this peculiarity, the most starchy and most sweet kernels were selected from a self-fertilized semi-starchy ear. The result of two years' selection of the most wrinkled kernels for planting produced ears nearly all of which were of a pure sweet nature. No ears have as yet been produced of a pure starchy nature. 
Such semi-starchy ears are often encountered by sweet corn canning factories and give much trouble. Selection of the most wrinkled seeds for planting will assist in eliminating the semistarchy types.

\section{Yellow-White Crosses}

Our results indicate that in many cases there are two separately inherited characters for yellow color in corn, either of which can produce this color. This fact, although of great scientific importance, does not materially change the methods of producing pure seed of either the yellow or white variety. The color which produces the yellow varieties of corn is an endosperm character. When white corn is crossed with pollen from a yellow variety the resulting progeny always has a yellow color, although it is sometimes lighter than the pure yellow. If the white corn plot is not completely isolated from the yellow variety some hybrid seeds will be produced. On saving the white corn seed, those seeds which are hybrid can be detected by their yellow color and removed, with the assurance that when nothing but white seed is planted the seed is pure for this character.

When yellow is the female parent and is crossed with white corn the resulting seed is yellow. In some crosses the hybrid seeds are light yellow, but, on the other hand, some hybrid yellowwhite seeds are dark yellow, so it is impossible to separate the pure yellow from the hybrid yellow seed except by breeding. Therefore, yellow corn, when intended for seed, must be grown at a considerable distance from white varieties.

Intentional crossing of two varieties is usually done with the idea of obtaining an improved variety by combining the desirable qualities of both parents. Suppose a white corn with a large stalk has been crossed with a yellow variety with a good ear and that a type has been produced with both these characters (large stalk and good ears), but the ears are composed of such a heterogeneous mixture of yellow and white seeds that they have no value as seed corn. The problem is to obtain ears which will produce either all white or all yellow seeds. The easiest method is to select only pure white seeds for planting, which in turn will produce only white corn. If a yellow variety is desired it can be most easily produced by planting all yellow seeds and self- 
fertilizing a number of ears, i. e., the pollination of the sillss of a plant by its own pollen grains. This is most easily performed as follows:

Just before the silk appears a bag should be placed over the ear and another over the tassel. About five days later, when the silk is well showing, the stalk should be bent over and the tassel bag carefully removed. This will contain a certain amount of yellow dust or pollen which should be carefully dusted over the silks, and the ear again covered. After maturity, these handfertilized ears should be harvested and examined. All ears which contain only yellow seeds will produce only yellow progeny. It is necessary to self-fertilize a large number of ears so that several pure ears may be allowed to cross naturally the following season in order not to obtain evil effects from inbreeding.

\section{Purple-White Crosses}

Only a few races of purple corn are grown in Connecticut, of which Black Mexican sweet is the best known example, although a purple pop variety is sometimes seen in the market. This purple color is due to a dye which is present in a single row of cells, known as the aleurone layer, which is found just underneath the pericarp or outer hull of the seed. As this layer is a part of the endosperm, there is an immediate effect when nonpurple races are crossed with purple varieties. The conditions, however, are different than in the crosses previously mentioned.

From an analysis of crosses between Black Mexican sweet and several non-purple races the following facts have been established. The purple color is due to at least two separately inherited factors found in the reproductive cells, both of which must be present in order to produce a purple condition. By crossing a white race which we found to contain one of these factors with another white race which contained the other factor, purple seeds were obtained. In one cross there was no visible effect in some kernels when a non-purple race was crossed with pollen from a purple variety. This we explain by the presence of something in this race which inhibited the production of purple. These facts, however, will not be further discussed here.

When pure seed of a purple race is desired, it is necessary that it be isolated from other corn plots. Of course, when Black 
Mexican sweet is fertilized with pollen from starchy races, either dent, flint, or pop, those seeds which are of a hybrid nature will be of a hard starchy condition and can therefore be rejected.

Non-purple races, of either flint, dent, pop, or sweet, from which seed is saved, should not be grown near a purple variety, as there is no surety that hybrid seeds can be rejected. All kernels showing any purple color will be found to be hybrids and by rejecting these the greater part of the hybrid seeds can be removed.

\section{Red Pericarp-White Pericarp Crosses}

The pericarp of corn is the outer hull. It is in this portion of the seed that the red color of the common red races of corn is found. If a paper bag be placed upon an ear before silking time and be removed after the silks have fully developed, thus leaving it unfertilized, the places on the ear where the seeds would have been formed, had pollination taken place, will be found to consist almost entirely of this outer hull or pericarp. This portion of the seed is therefore just as much a part of the mother plant as its tassels or silks and is not immediately affected by pollination. For this reason an ear has either a red pericarp color on all of its seeds or on none of them.

If a seed is soaked for a short time in water this outer hull may be easily removed. This red color conceals all other colors which may be present in the seed. Thus, an ear of corn may contain both yellow and white, or purple seeds, or any of these colors in a pure condition, yet if the red pericarp is present these facts are obscured. Thus we are further impressed with the fact that all of the characters so far discussed are separately inherited.

If a red pericarp corn is crossed with a non-colored race, no matter which is the female parent, there will be no immediate visible effect. If these hybrid seeds are planted, the crop for the following season will consist of all red pericarp ears. If these ears are self-fertilized and grown, red and non-red pericarp ears will be received in the ratio of three to one.

Besides this solid red pericarp there are mosaic red, commonly called "Bloody Butcher," varieties. This mosaic red is inherited as a pattern color. In common with many variegated races of 
plants this condition does not breed true but always gives some non-colored and, in some cases, some deep red progeny. There is also a pericarp color which varies in intensity due to light conditions. If the ear is stripped of its husks before maturity all seeds are partially covered with red, in other cases the red color is only seen as a slight blush on some seeds near the tip of the ear. This color is inherited in the same manner as the deep red pericarp color.

As there is no method of detecting a cross between red and non-red races, seed of either sort should only be grown in isolated plots at some distance from the other variety.

\section{Red Cob-White Cob Crosses}

The directions for selecting exhibition corn, issued by the extension department of the Massachusetts Agricultural College, state that white corn usually has white cobs, that red cobs in yellow corn are preferred, while a variation in cob color shows a mixture and poor breeding.

It is not believed that the color of the cob is of any practical importance, although for the sake of a uniform appearance a "mixture" is not advisable. In a study of a cross between a red pericarp, red cobbed variety, and a race which lacked these colors, the pericarp and cob colors were coupled in inheritance, i. e., when separation took place all red cobs had red pericarps and all white cobs had white pericarps. In other cases no coupling has been observed and each character is separately inherited.

The cob color behaves exactly similar to the pericarp color in crosses. Red is dominant to white and in the second generation there are, on the average, three red cobs to one white.

In case of a mixture of red and white cobs, or pericarp colors, pure races for either the one or the other can be most quickly got by self-fertilizing a number of ears, as explained under the heading Yellow-White Crosses. All non-colored self-fertilized ears for either cob or pericarp will give non-colored progeny. Of the self fertilized red ears, one-third will breed true and twothirds will again give a mixture. The breeding nature of these can be tested by growing about ten hills of each and noting results. The remainder of the seeds from each ear should be reserved and all ears which, by test, give only red-eared progeny can be used to multiply the variety the following year. 


\section{Summary of Crosses Showing Dominance}

In the preceding discussion we have considered the appearance of crosses, the production of seed of pure varieties, and the inheritance of characters.

Summing up these results, we find that when a white corn is crossed with a yellow variety the resulting seeds are always yellow. Likewise when sweet corn is pollinated with starchy races (dent, flint, or pop) the resulting seeds are always of a starchy appearance. Pure seeds for the characters, sweetness, or white color can therefore be told by inspection. When ears show a mixture of yellow and white or starchy and sweet seeds we may be sure that the white seeds and the sweet seeds are pure for these respective characters. Thus no extreme isolation from other varieties of either sweet or white corn seed plots need be practiced, as the crossed seeds can be detected and eliminated. Seed plots in all other cases must be isolated from other varieties.

In case a mixture has been received of yellow and white seeds and a yellow race is desired, this can be obtained by planting yellow seeds and self-fertilizing a number of ears, as we know that all self-fertilized ears which contain only yellow seeds will thereafter give yellow progeny.

When a variety produces some colored and some non-colored ears for either cob or pericarp colors, there is no surety that either selected color will breed true. As a larger part of the seeds of an ear is normally "cross-fertilized" there is a small chance of receiving either a red or white ear in which some seeds will not be fertilized with pollen from a plant bearing an opposite color and, when planted, give a mixture. Pure varieties can be most quickly obtained by self-fertilizing a number of ears as previously explained under the heading "Red Cob-White Cob Crosses:"

It is always necessary to self-fertilize a number of ears so that several pure ears may be allowed to cross naturally the following season.

When attempting to produce an improved variety by crossing two types, each of which contains a desirable character, the aim of the breeder is to combine both desirable characters in one variety. As we have learned, each character is generally sep- 
arately inherited, although some cases of coupling have been reported in which two or more characters are inherited together. The method of inheritance of any character can be determined by crossing a variety which contains this character with another in which it is either absent or is present in a modified condition and by studying the appearance of this character in later generations. The knowledge of the manner in which each character is inherited enables the breeder to combine desirable features of one variety with those of another.

When the parents differ by two separately inherited characters we may illustrate the results as follows:

Suppose one parent is a yellow sweet corn in which there is only one inherited factor for the yellow color and the other parent is a white flint corn. As we have seen, yellow is dominant to white, and flint or starchiness is dominant to sweetness. Since these are endosperm characters, the immediate cross of the above parents will be a yellow flint corn. But the following generation, if self-fertilized, will consist of ears containing four sorts of seeds in the ratio of nine yellow starchy, three white starchy, three yellow sweet and one white sweet. The yellow starchy corn is produced when the factors for yellow and starchiness are both present. As both are dominant factors, only a part of the nine yellow starchy seeds will breed true. Theoretically, one out of every nine will give ears containing only yellow starchy seeds, two will breed true for the yellow color but will be hybrid for the starchy character, two others will breed true for the starchy condition but will give both yellow and white seeds, and four will again produce ears containing all four sorts of seeds.

Of the three white starchy seeds, all will breed true to the white color, but only one of every three will give pure starchy ears. Likewise, of the three yellow. sweet seeds, all will give sweet progeny, but only one out of every three will give pure yellow ears. The white sweet seeds will all breed true, giving only white sweet progeny.

Our parent varieties were yellow sweet and white flint. From crossing these varieties two new sorts have therefore been produced, namely, white sweet and yellow flint. This cross illustrates what we mean by a recombination of characters. 


\section{Inheritance of Characters Which do not Show Dominance}

From the viewpoint of the improvement of corn by a cross between two varieties, each of which contains some desirable character, the phenomenon of dominance is of little importance. The result desired is to obtain a race which contains both beneficial characters.

The following crosses conform to the essential feature of Mendel's law, i. e., the separation of characters in the reproductive cells of hybrids and their chance recombination-although the conditions are of a complex nature.

After crossing, the characters given below do not show dominance. The first hybrid generation is intermediate in appearance and the second hybrid generation gives both intermediate and parent types.

Crosses between Flint and Dent races.

Crosses between races which differ in row numbers.

Crosses between races which differ in height of plants.

Crosses between races which differ in length of ears.

Crosses between races which differ in size of seeds.

\section{Crosses between Flint and Dent}

Flint corn has hard, smooth and oval grains generally nearly as broad as long, while dent corn is indented on the top and the length of the seeds is much greater than their breadth. The characteristic difference between flint and dent is due to the amount and position of horny starch in the endosperm. Flint seeds have the embryo and soft starch surrounded by the horny starch. In dent races the soft starch extends from the center to the cap, the sides of the seed being composed of horny starch.

There is no immediate visible effect when dent and flint races are crossed, the character being inherited as a plant character. For this reason seed of either sort must be produced in isolated plots. Frequently an ear from a flint-dent cross contains both indented and flinty seeds, yet selection of either kind for planting gives like results. An ear which contains both flint and dent seeds is generally a hybrid.

The first generation of a cross between a flint and dent is of a uniform nature and intermediate in appearance. Sometimes this generation is more like the flint parent, sometimes more like dent 
in appearance and in other cases strictly intermediate. Selffertilized ears of this generation produce a wide range of variation the following season, a few ears resembling flint, others resembling dent, and the greater part again of an intermediate nature. Self-fertilized ears either of the pure dent or pure flint type produce pure races when grown, but the intermediates again show wide variabilities. In this cross there is often difficulty in separating pure dent or pure flint from intermediates, by inspection. As in some previous cases, the only sure method of determining the breeding nature of an ear is by growing it and examining its progeny.

\section{Crosses between Races which Differ in Row Numbers}

There is a wide range of row classes in corn. The most common flint races are eight-rowed sorts and some dent races produce as high as thirty-six rows. This character is quite markedly affected by conditions; thus, an eight-rowed flint frequently produces some twelve-rowed ears. A sixteen-rowed selection which has been inbred for a number of years has a normal fluctuation of from twelve to twenty rows with a mean value of sixteen rows. In pure races these fluctuations, due to environmental or physiological conditions, are non-inherited.

Crosses between races which differ in row numbers are of an intermediate nature in the first generation, the range of classes being somewhat larger in the second generation than in the first. Some of these second generation plants breed comparatively true, although the greater part again break up the following year.

There must be several inherited factors for row classes in corn, as different varieties breed true to different row classes. The row classes of most commercial varieties of corn can be changed by selection and either increased or decreased. This is due to the fact that corn is naturally cross pollinated, and a commercial variety really consists of a complex hybrid condition.

\section{Crosses Between Races which Differ in Height of Plants}

Plant height is a complex character and, without doubt, due to many inherited factors. Our reasons for this belief are the large number of known varieties which differ from each other in plant height. In common with other size characters, environmental 
conditions have the power to greatly modify the height of plants. The fluctuations within a pure race are very large and make the analysis of the inheritance of such characters almost impossible. Some of our experimental races have been constantly inbred for a period of six years and are very uniform in appearance. Under ordinary field conditions, the difference in height of different plants of such pure races is often as great as two and a half feet.

Two crosses have been studied between races which differ in plant height, both giving similar results. One was a dent-flint cross and the other a cross between Tom Thumb pop and Black Mexican sweet. The results were as follows: The first generation of the cross proved to be as uniform as either parent and was somewhat larger than the average of the parents. This increased height over the average of the parents is due to increased vigor and not to dominance.

Self-fertilized ears of this first generation, when grown, produced a wide range of variability for plant height, embracing the range of both parent forms. Of course, it is not to be expected that many of these forms will breed true the following year, yet selection of forms like either parent will doubtless give an approach toward the parental condition.

\section{Length of Ears and Size of Seeds}

Only one cross between races which differ in ear length has been carefully studied. This was between Tom Thumb pop, with an ear length of from five to eight centimeters, and Black Mexican sweet, with an ear length of from thirteen to twenty-one centimeters. The first generation of this cross had a range of variation of nine to fifteen centimeters. This generation was no more variable than the parent races, and was of an intermediate ear length, although somewhat larger than the average of the parents. The second generation was very variable and produced some ears which closely approached the parental forms in length.

The above cross was also used to study the inheritance of weight of seeds. The size of the seeds is determined in a large measure by the pericarp, which is a mother plant character. For this study, twenty-five seeds were weighed from each ear. The first generation was intermediate in weight of seeds and no more 
variable than the parent forms. The second generation presented a wide range of variation. Some short ears produced large seeds, while some long ears produced small seeds.

\section{Summary of Crosses not Showing Dominance}

The preceding crosses do not show the phenomenon of dominance, yet all behave in a similar manner. The first generation of these crosses is of an intermediate appearance and the second generation has a range of variation from one parent form to the other. Some of these second generation forms will breed true, but it is impossible to determine, by inspection, which these are. The only method of determining the breeding nature of these second generation forms is by row tests.

\section{Abnormalities}

There are a number of abnormalities of corn which are often found in commercial races. These appear to be of two sorts; first, those which are produced by some unusual environmental condition and are non-inherited, and second, inherited abnormalities. Of these two classes the heritable one is of interest to the commercial grower or seedsman because it is important to know the quickest method of getting rid of these abnormalities if they appear.

The following abnormalities have been found to be inherited:

Dwarf forms.

Divided ears.

Irregularity of rows of seeds.

\section{Dwarf Forms}

Dwarfs have appeared several times in our cultures, and in all cases but one in strains which have been inbred for one or more seasons. Inbreeding, as used here, means the pollinating of the silks of a plant by its own pollen grains.

In one instance, some divarf plants appeared in a commercial culture of Stowell's Evergreen Sweet. A normal plant from this culture was inbred and gave both dwarf and normal plants the following season. A cross was made between a normal plant and a race in which dwarfs had never appeared, which, when grown, 
gave only normal plants, two of which were self-fertilized. One of these ears produced only normal plants, and the other gave both normal and dwarf forms.

Several attempts to self-fertilize such dwarfs have proved unsuccessful and in one case when a few mature seeds were produced they failed to germinate. Emerson of Nebraska succeeded in self-fertilizing some similar dwarfs which appeared in his cultures and when grown only dwarfs were produced.

It seems very probable that we are dealing with a condition in which normal plants are dominant to dwarfs, as is the case of the tall and dwarf peas. The dwarfs which have appeared in our corn cultures, unlike dwarf peas, are absolutely valueless. The low per cent. of seeds which germinate from strains which produce some dwarfs points to the fact that in some cases such abnormalities have not been able to develop.

If dwarfs appear in an otherwise valuable strain they can be most quickly eliminated as follows: By hand pollinating a large number of ears we may expect to receive one out of every three which will give only normal plants. The hand pollinated ears should be tested by planting a part of each and reserving the remainder of the seeds. The seed from all ears which in this test gives only normal plants may be used to develop the strain the following season. Such a method should not be used unless the strain is a valuable one.

\section{Divided Ears}

A desirable ear of corn should be cylindrical in form and should not have too large a cob, i. e., the proportion of shelled corn to cob should be large.

There are several classes of abnormalities which show different degrees of division of the cob. A form with a monstrous, flattened, and in extreme cases a divided tip, is frequently seen in races which have a large number of rows, such as dents and pops. At our State Fair this year, one entry for the best six-ear lot of yellow dent corn contained one such ear. Such an ear produces a large proportion of cob which is valueless and also an ear which is hard to shell.

The above abnormality belongs to the inherited class, although environment has the effect of retarding or accelerating its mani- 
festation. One of our inbred races of dent produces, on the average, about two-thirds of its progeny with a flattened ear and one-third with both a flattened ear and a divided tip. A cross between this strain and a strain with a flattened cob gave, in the first generation, about the same per cent. of divided tip ears as is produced by the divided tip parent.

Divided tip is dominant in crosses to normal tip, but the dominance is not complete. In such cases there is the added difficulty of not being able to tell the recessive form from the dominant by inspection. Flattened cobs have proved dominant in crosses over cylindrical cobs.

There are different degrees of cob division and in the extreme case the cob is grooved and only four rows are produced. Although we have not found this four-rowed condition to be inherited, there is no doubt of the fact that the tendency to division is sometimes inherited. We have often found the same plant producing one ear which is four-rowed and another with eight rows. When the tendency to division is an inherited character an inspection of the eight-rowed ear showed a tendency towards row splitting at the butt of the cob. In some cases we found this character to be non-inherited. Any row culture producing over five per cent. of such ears should probably be discarded.

\section{Irregularity of Rows of Seeds}

The greater part of the ears of our commercial varieties of corn have regular rows. Sometimes, as we have noted, two or more rows are dropped from a part of the ear, but even then the rows of seeds are regular and straight.

Nearly all varieties produce some ears which show irregular rows, the kernels being packed closely together on the ear, making it almost impossible to count the row number. Such irregular ears are undesirable, the seeds presenting differences of size and shape which are not conducive to even dropping by cornplanters. Regular-rowed ears also present a more attractive appearance.

Country Gentleman sweet corn is an irregular-rowed variety which proves that in some cases irregularity of rows is an inherited character. Irregular-rowed ears have often appeared 
in our cultures and proved to be non-inherited, in all but one case. A self-fertilized, irregular-rowed ear of a white flint strain produced thirty-three normal and fifteen irregular ears, while a normal self-fertilized ear produced one hundred and twenty-five normal and five irregular ears. One of these five irregular ears was self-fertilized, and the following year produced fifty-six regular ears and one irregular ear. A regular-rowed ear from the irregular-rowed strain produced seventy-nine regular and twentynine irregular-rowed.

The manner of the inheritance of this character cannot be definitely stated, yet the character is an inilerited one. Any commercial row culture which contains much over five per cent. of irregular ears should be discarded to eliminate the undesirable character.

\section{Summary of Results}

Corn is a normally cross-fertilized plant, and for this reason a commercial variety is composed of many types. Any variety can be made more uniform by selection. The benefit of selection seems chiefly due to the elimination of the poorer types, leaving only the better sorts.

The improvement of corn by selection has been thoroughly discussed in previous bulletins and will be only briefly mentioned here. Bulletin $\mathbf{I}^{2} 2$ gives directions for producing a breeding plot in which each row is grown from a single ear. Each row is then harvested separately, the yield and appearance determining the value of the selection. Such commercial row cultures have been and will continue to be of great value. We should not, however, expect too much from such a method. The increased yields which have been received by such methods are believed to be due to the elimination of undesirable types of which the preceding abnormalities are examples. Row cultures are of inestimable value in such work.

In Bulletin 168 we discussed corn breeding methods and came to the conclusion that the growing of first generation hybrids would prove beneficial and materially increase corn yields. Some varieties will doubtless prove more beneficial for this work than others; however, all investigators agree that crosses between highly selected sorts will prove most valuable. 
It is hoped that the corn growers of Connecticut will not discard this article because of its technical nature and the difficulty of understanding this class of results. The subject of the inheritance of plant characters is a complex one, yet the seedsman or farmer who applies the known principles of inheritance and breeding to his own work will certainly receive a benefit thereby. 



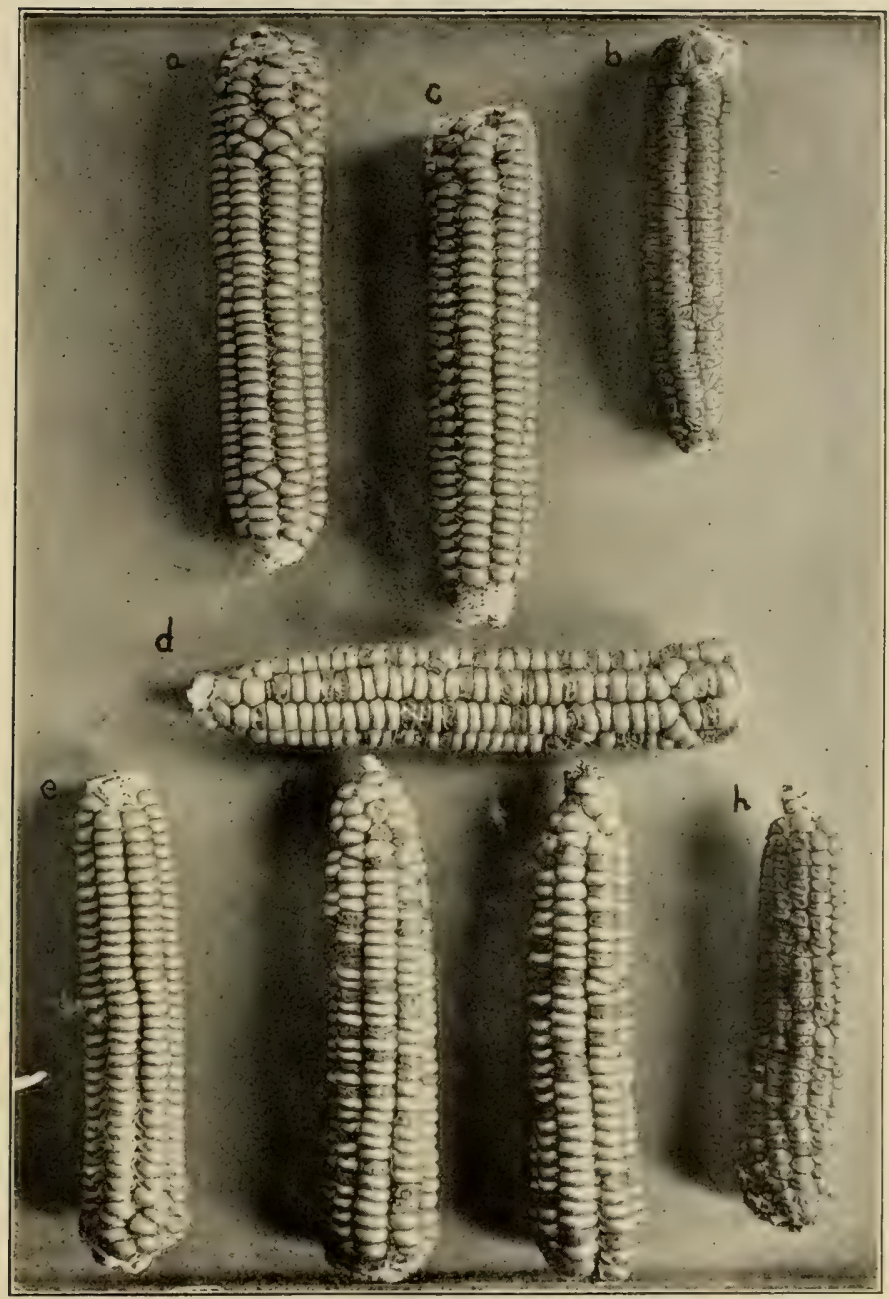

$a$, Rhode Island white flint (starchy parent); $b$, Early Crosby (sweet parent); $c$, result of immediate cross of $a$ and $b$, showing dominance of the flint type; $d$, result of planting seeds of $c$. The result of planting starchy seeds of $d$ is shown by $\mathcal{c}, f, g$. The result of planting sweet seeds is shown by $h$. 



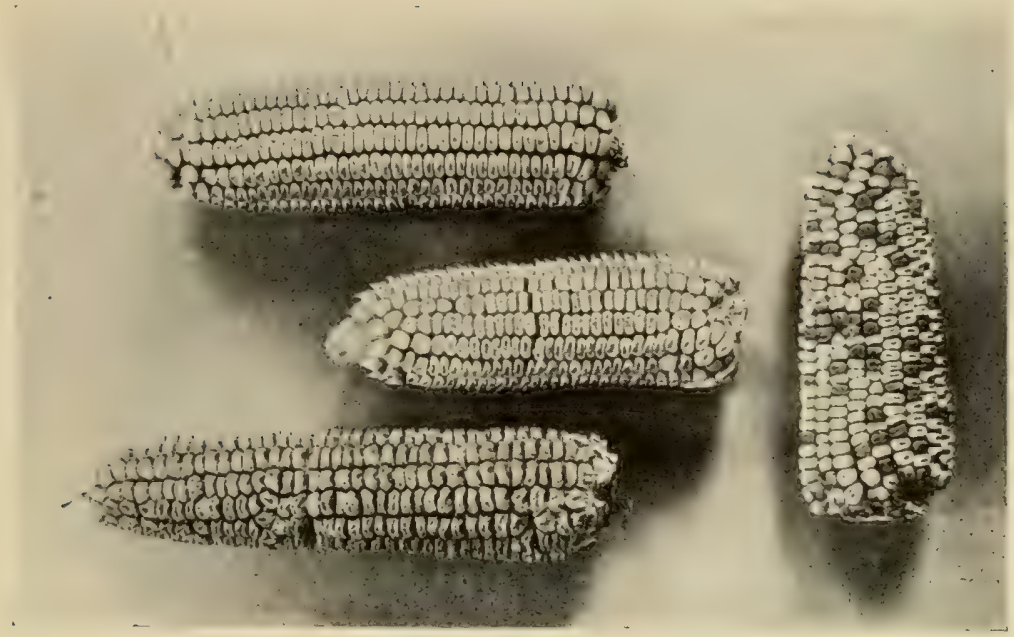

$a$, At left, upper ear, Illinois low protein dent. Middle ear, immediate result of cross between low protein dent and Stowell's Evergreen swect, and lower ear, Stowell's Evergreen sweet. At right, result of planting seeds of the hybrid ear.

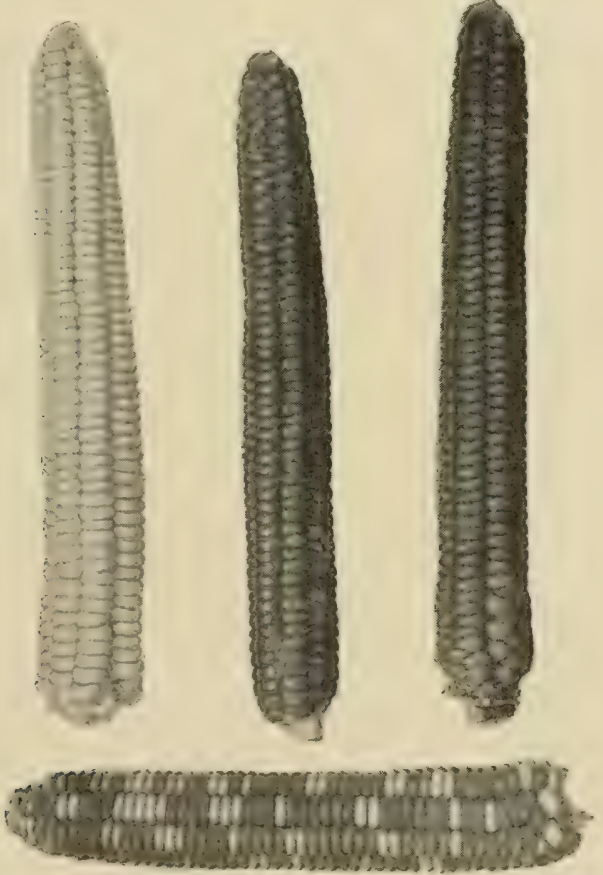

b, At left, Rhocle Island white flint; at right, Longfellow yellow flint; in center, immediate result of cross between yellow and white flint, showing dominance of the yellow color. Lower ear is a self-fertilized ear grown from hybrid seeds of central ear. 


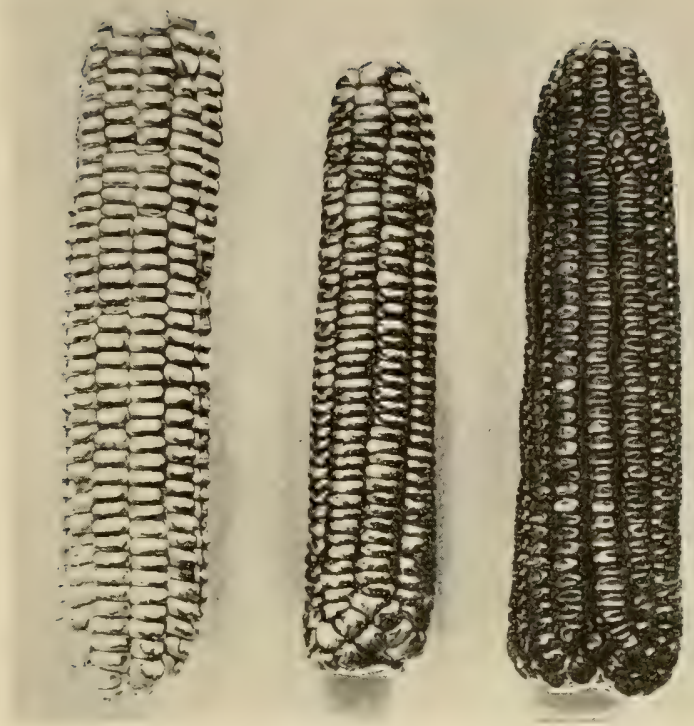

$a$, At left, the color which develops in sunlight; in center, variegated color which does not breed true; at right, common red pericarp.

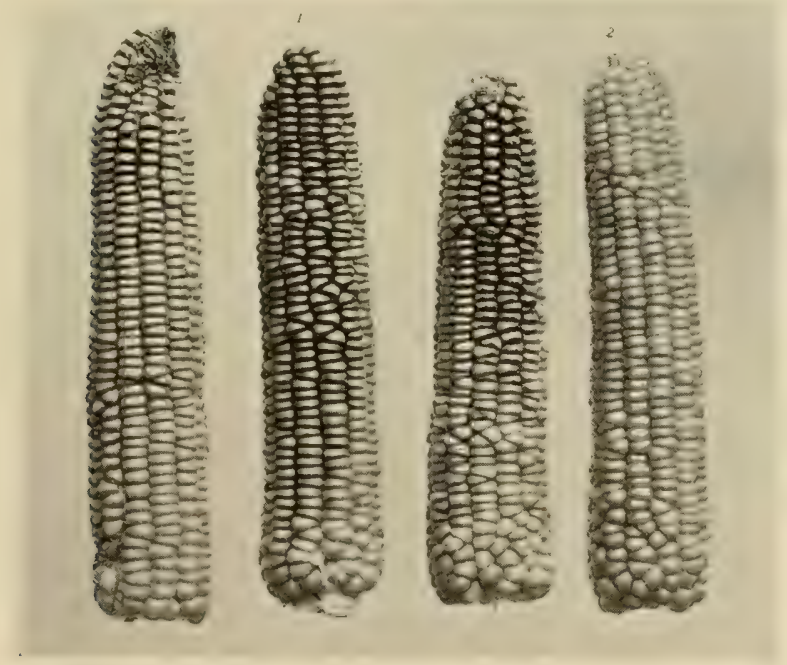

$b$, The second generation of a cross between the pericarp color which develops in the light and a white pericarp variety, giving, on the average, 3 colored ears to I white. The non-colored ear, if self-fertilized, will breed true. One out of every three, on the average, of self-fertilized colored ears will breed true. 



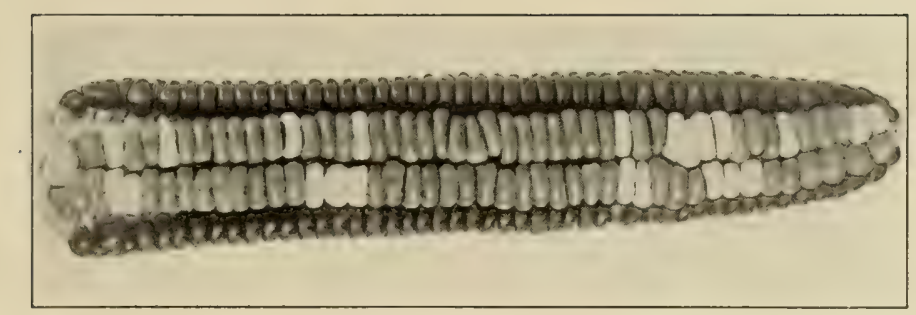

$a$, The first generation of a cross between a red and white pericarp, showing dominance of the red pericarp. The pericarp has been removed from two rows of seeds, showing a mixture of yellow and white endosperm colors.

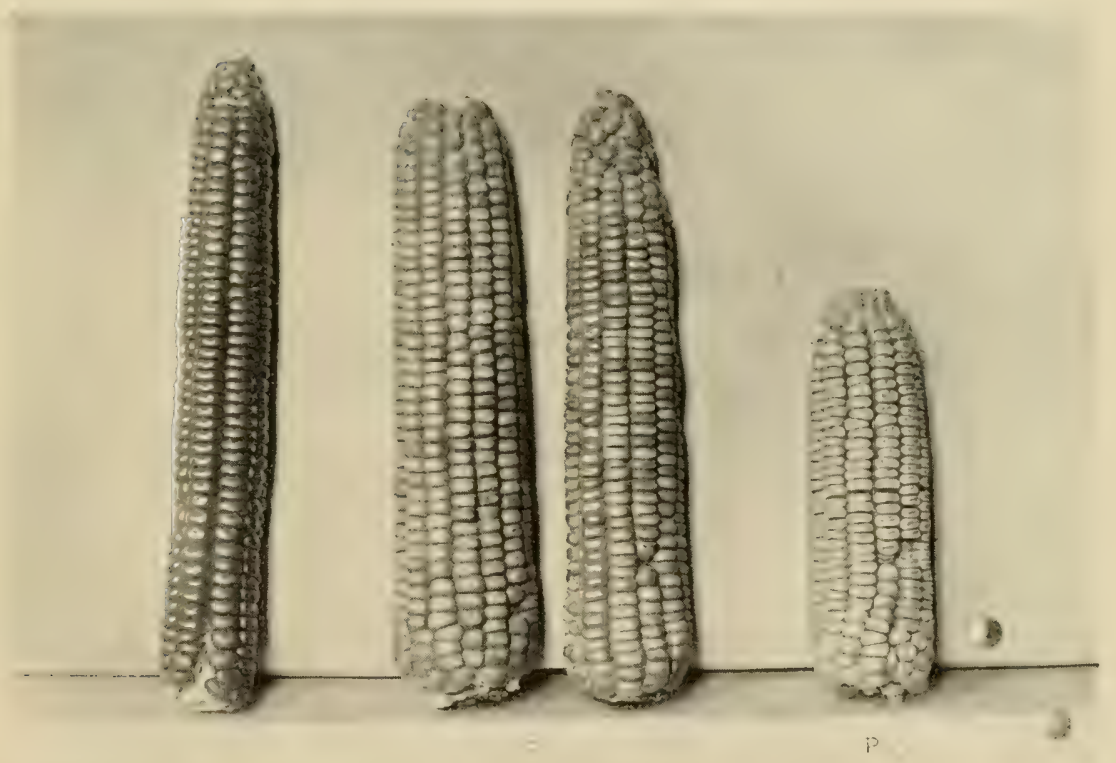

$b$, At left, Longfellow flint; at right, Illinois high-protein dent; in center, result of growing a cross between them. 



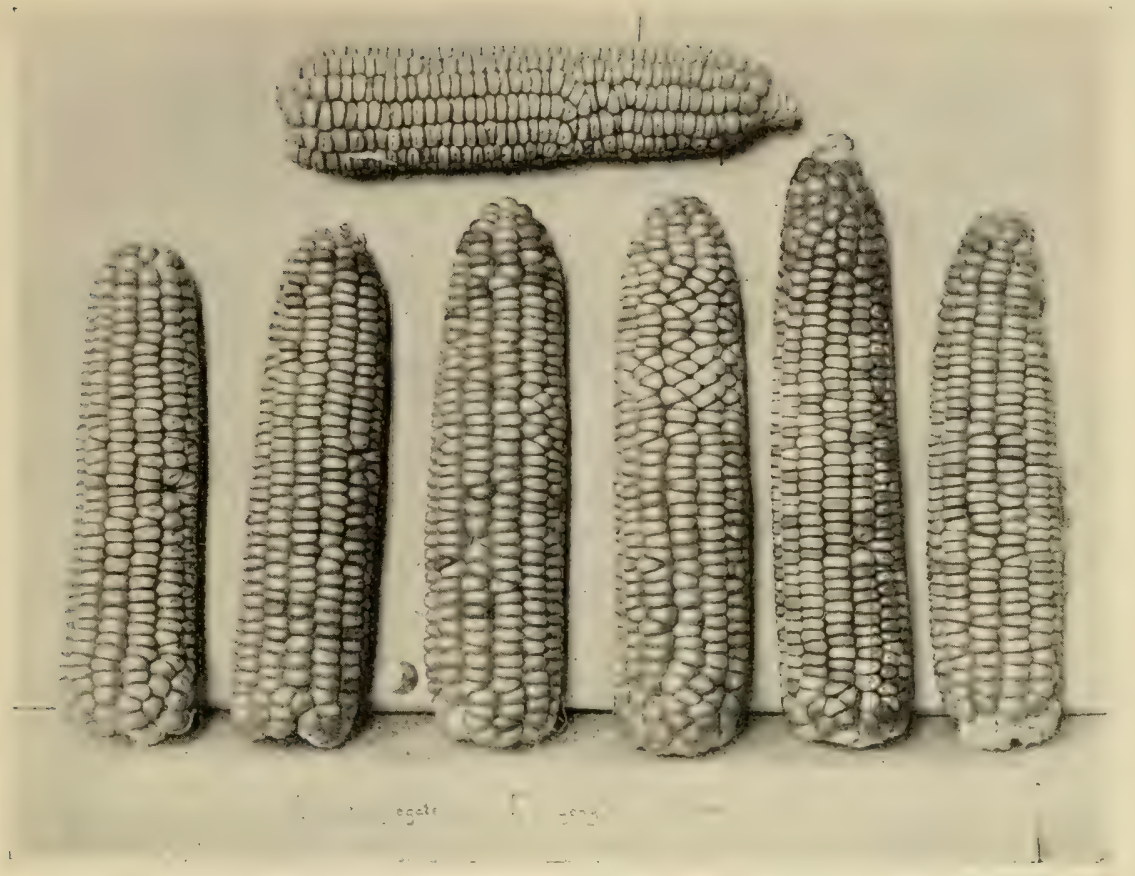

$a$, The ear above is a self-fertilized dent ear, received from growing the hybrid shown in Plate IS, b (frequency alout I in Io). Random sample of its progeny below.

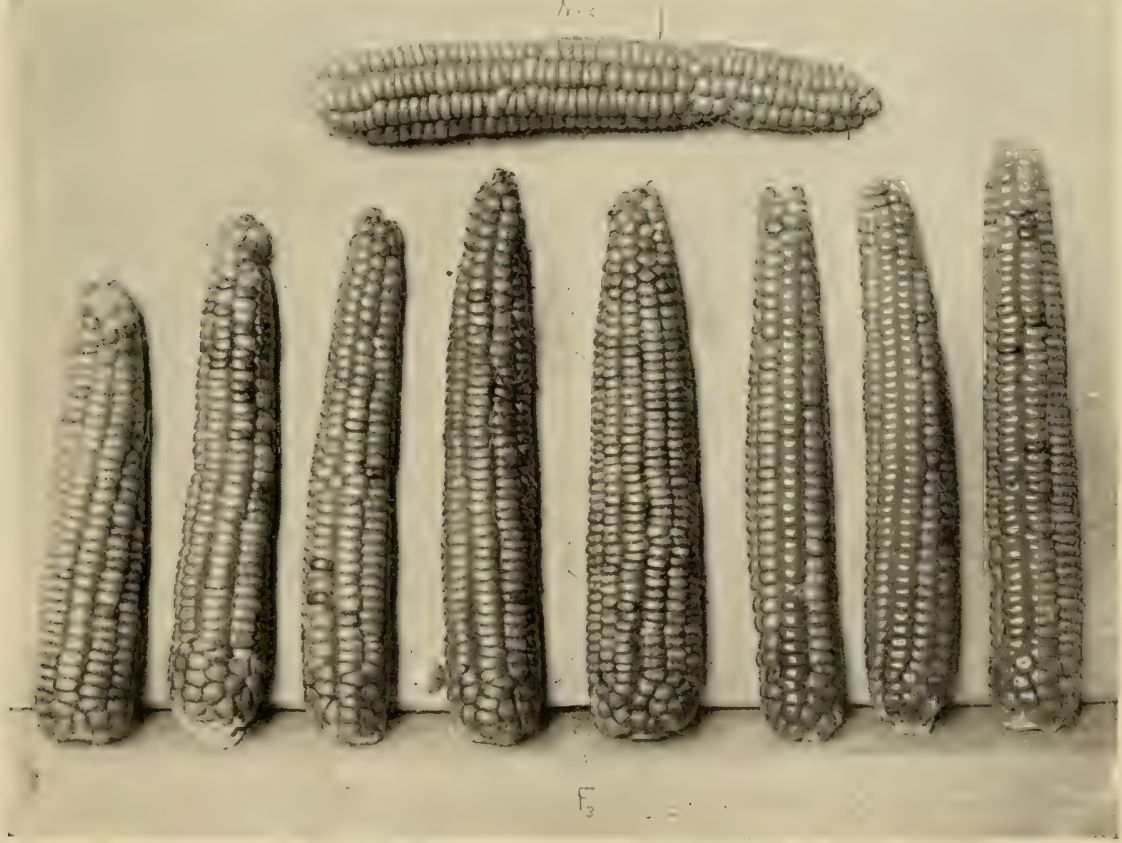

$b$, The ear above is a self-fertilized flint ear, received from growing the liybrid shown in Plate IV, $b$ (frequency about I in I6). Random sample of its progeny below. 



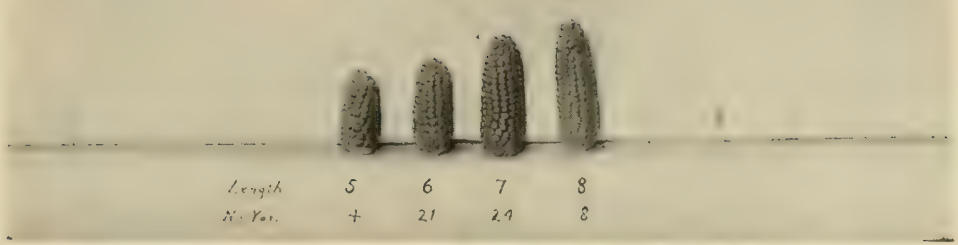

$a$, Tom Thumb pop, showing variation in length of ear. Length is given in even centimeters and the number of individuals in each class is given below (one-sixth of natural size).

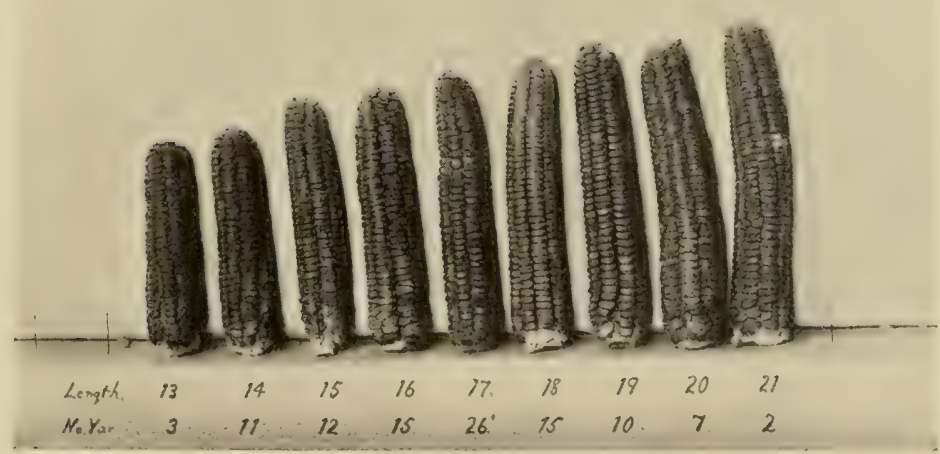

$b$, Black Mexican sweet, showing variation in length of ear (onc-sixth of natural size). 



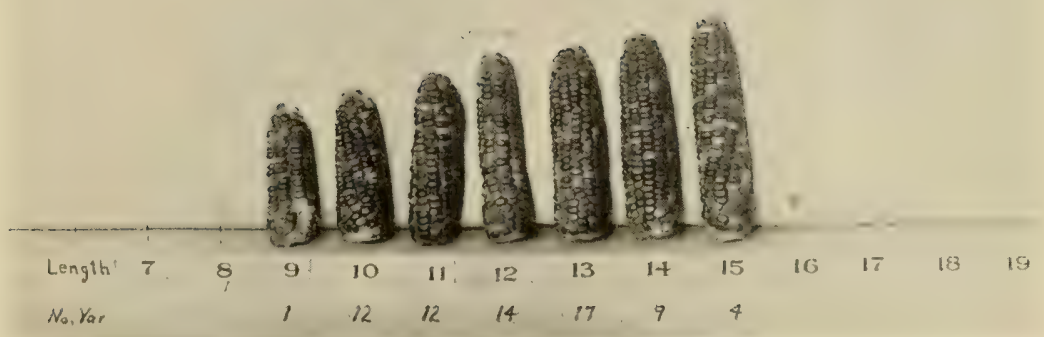

$a$, Variation in length of ear of the first generation of a cross between Tom Thumb and Black Mexican (one-sixth of natural size).

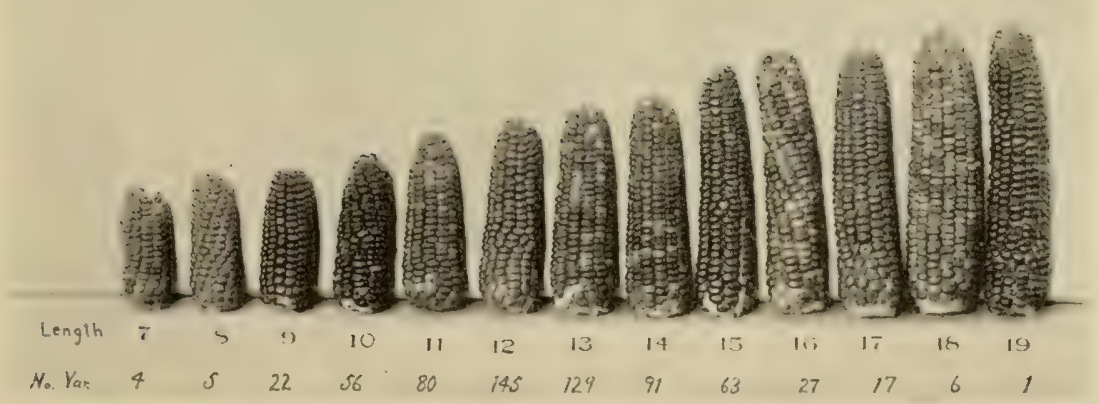

$b$, Variation in length of ear of second generation of cross between Tom Thumb and Black Mexican (one-sixth natural size). 



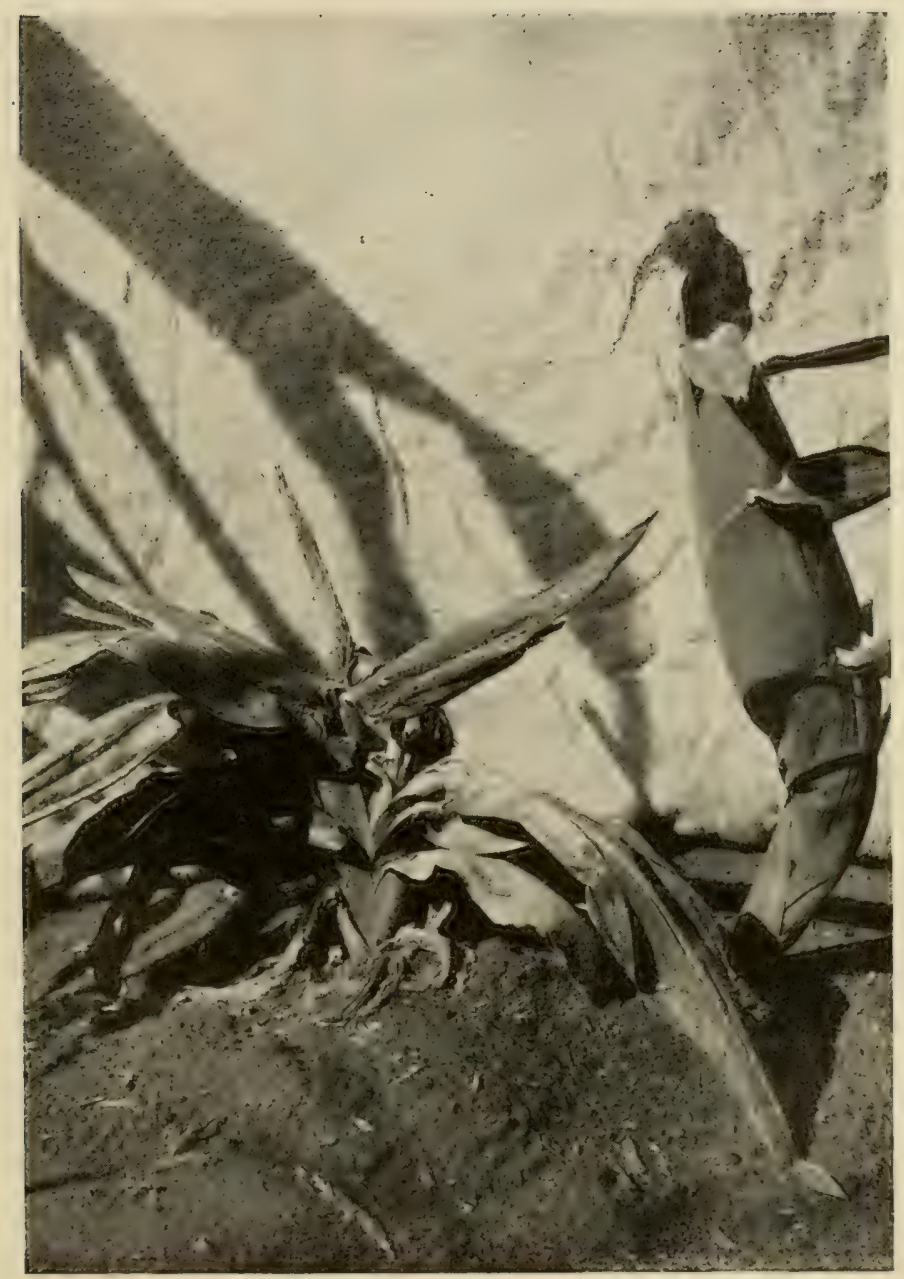

A dwarf, appearing in a commercial culture of Stowell's Evergreen sweet, compared with a normal ear of the latter. 



\section{INDEX.}

PAGE

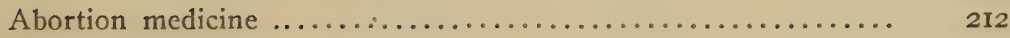

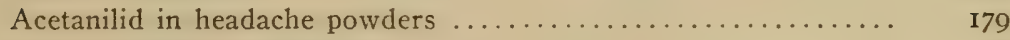

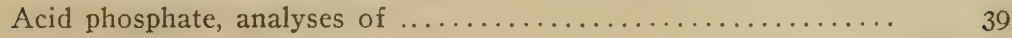

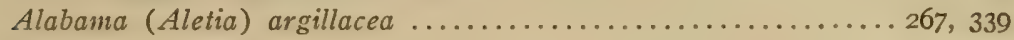

Aletia argillacea ................................. 339

Alfalfa, notes regarding yield of $\ldots \ldots \ldots \ldots \ldots \ldots \ldots \ldots \ldots \ldots \ldots \ldots \ldots$

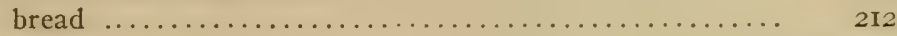

meal .................................... 2270

American Agricultural Chemical Co.:-

A. A. C. Co.'s Complete manure with $10 \%$ potash ....... 62

Complete tobacco manure .................... 62

(Carb.) $\ldots \ldots \ldots 62$

Grass and lawn top dressing .......... 62

H. G. Fertilizer with 10\% potash ....... 62

Tobacco manure .............. 62

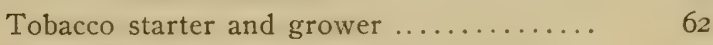

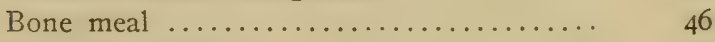

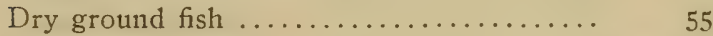

Fine ground bone ............... 46, 48

Grass and oats fertilizer .......... 54, 56

Ground tankage 6 and $30 \ldots \ldots \ldots \ldots \ldots$....... 50

Acid phosphate ................. 40, $4 \mathrm{I}$

Castor pomace ................... 37

Double manure salt ............... 43

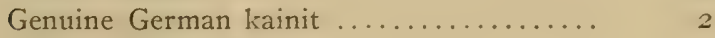

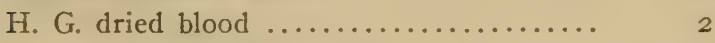

sulphate of potash $\ldots \ldots \ldots \ldots \ldots \ldots \quad 2$

Muriate of potash ............... 44

Nitrate of soda ................. 23

Thomas phosphate powder ............ 38

Bradley's Complete manure for potatoes and vegetables .. 62

top dressing grass and

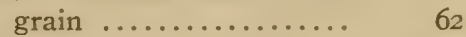

with $10 \%$ potash $\ldots \ldots \ldots \ldots .62$

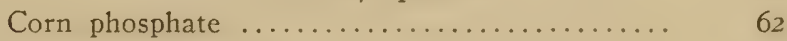

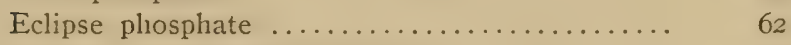

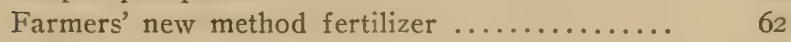

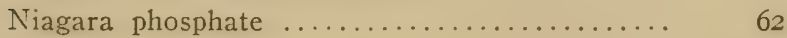

Potato fertilizer ..................... 62

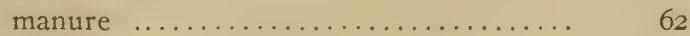


American Agricultural Chemical Co., cont'd:-

Page

Bradley's X. L. Superphosphate .................. 62

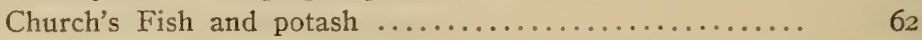

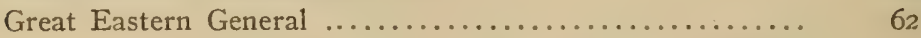

H. G. Vegetable, vine and tobacco fertilizer 62

Northern corn special ................ 62

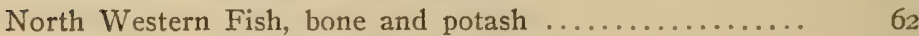

Market garden phosphate ............ 62

Potato and truck guano .............. 2

I0\% potato fertilizer ............... 6

Universal fertilizer ................. 62

Packers' Union Animal corn fertilizer ............... 64

Gardeners' complete manure .......... 64

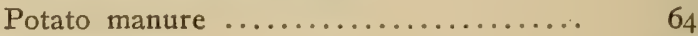

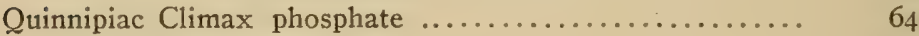

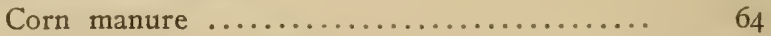

Market garden manure ................. 64

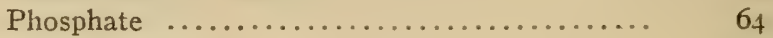

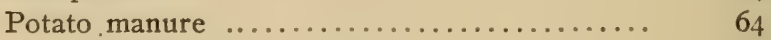

phosphate $\ldots \ldots \ldots \ldots \ldots \ldots \ldots \ldots \ldots .6 \% \ldots$

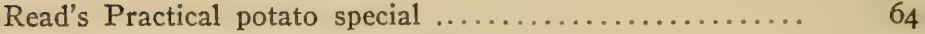

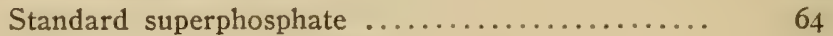

Vegetable and vine fertilizer ............... 64

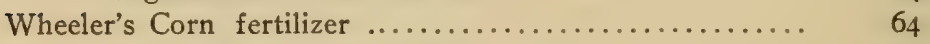

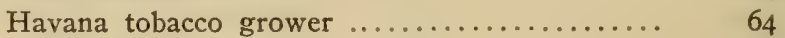

Potato manure ...................... 64

Grass and oats ....................... 54, 56

Williams \& Clark's Americus Ammoniated bone superphosphate ............. Corn phosphate ......... H. G. Special fertilizer . . Potato manure ..........

Potato phosphate .................

Royal bone phosphate ............

Anthracnose of cherries and plums ...................... 408

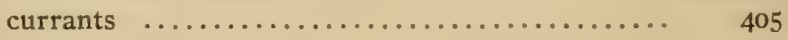

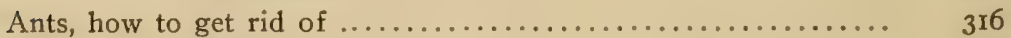

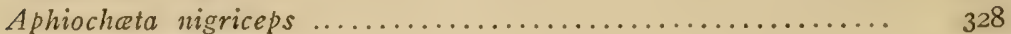

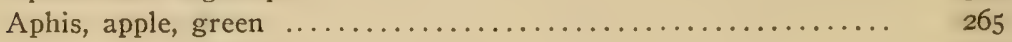

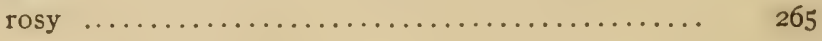

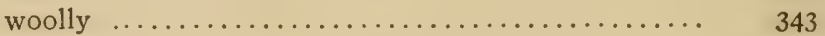

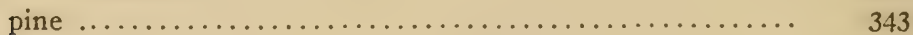

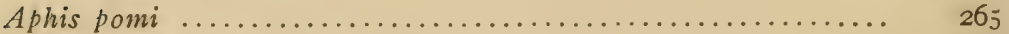

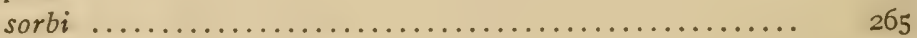

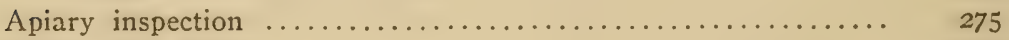

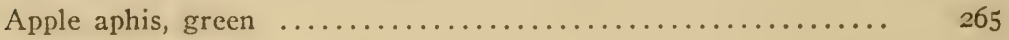

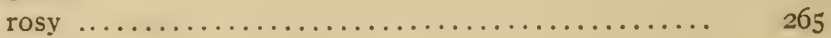


Apple aphis, woolly

Page

leaf crumpler ............................... 34

tent caterpillar .............................. 342

Apples, benefits of spraying ........................ ${ }_{36}$

candied ................................. ${ }_{20} 202$

details of spraying experiments ................ 376

immune and susceptible varieties ................ 359

leafburn .................................. 360

recommendations for spraying ................ 370

results of two years' experiments ................ 358

russeting $\ldots \ldots \ldots \ldots \ldots \ldots \ldots \ldots \ldots \ldots \ldots \ldots \ldots \ldots \ldots \ldots \ldots \ldots,{ }_{36}$

scald of ................................. $\quad 363$

spray injuries ............................ 360

Archips cerasivorana .............................. 309

Armour Fertilizer Works:-

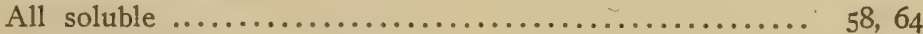

Ammoniated bone and potash $\ldots \ldots \ldots \ldots \ldots \ldots \ldots \ldots \ldots \ldots \ldots \ldots, 58,64$

Bidwell's Formula for all crops ................. 58, 66

Bone, blood and potash ........................ 66

Complete potato ............................ 58,66

Corn king ................................. 66

Fish and potash ............................ 58,66

Fruit and root crop special ................... 58,66

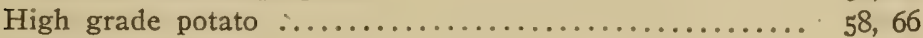

Market garden .............................. 66

Bone meal ................................ 46

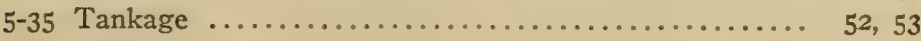

German kainit $\ldots \ldots \ldots \ldots \ldots \ldots \ldots \ldots \ldots \ldots \ldots \ldots \ldots \ldots \ldots \ldots, 43$

Nitrate of soda ............................ ${ }_{23}$

Star phosphate ............................. 40, 4I

Aspidiotus perniciosus .............................. ${ }_{26} 68$

Atlantic Fertilizer Co.:-

Atlantic Dissolved phosphate .................. 40, 4I

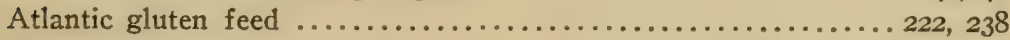

Atomic sulphur ................356, 36r, 369, 373, 375, 400, 402, 405

Baker Caster Oil Co.:-

Pure castor pomace ........................... 37

Barberry hedges, a pest of ....................... ${ }_{29} 292$

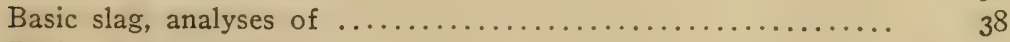

Basilona imperialis ................................ $34 \mathrm{I}$

Beef scrap ....................................... 254

Beet pulp, dried ................................... 2250

molasses ................................. 2250

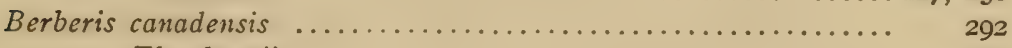

Thunbergii .............................. 292

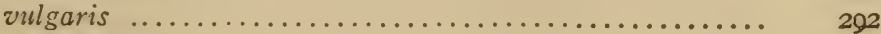


Berkshire Fertilizer Co.:-

Berkshire Ammoniated bone phosphate ................

Complete fertilizer .......................

Grass special

Long Island special fertilizer $\ldots \ldots \ldots \ldots \ldots$

Potato and vegetable phosphate $\ldots \ldots \ldots \ldots \ldots$.

Tobacco special (carb.) ................... 66

Ground bone ......................... 46

Black knot of cherries and plums .................... 399

Blatchford's calf meal ......................... 229, 252

Blissus leucopterus ................................ $\quad 265$

Boardman, F. E.:-

Boardman's Complete for potatoes and general crops ..... 66

Bohl, Valentine:-

Self recommending fertilizer .................... 46

Bone, method of valuation $\ldots \ldots \ldots \ldots \ldots \ldots \ldots \ldots \ldots \ldots \ldots \ldots \ldots$. 7

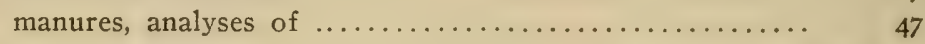

Bordeaux mixture .....................350, 361, 362, 369, 397

Bowker Fertilizer Co.:-

Bowker's Bone and wood ash fertilizer ............... 66

Complete alkaline tobacco grower ............ 66

Corn phosphate $\ldots \ldots, 66$

$($ carb. $\ldots \ldots \ldots, 66,82$
$\ldots \ldots$

Early potato manure $\ldots \ldots \ldots \ldots \ldots \ldots \ldots \ldots \ldots . \ldots \ldots$

Farm and garden phosphate .............. 66

Fisherman's brand fish and potash ........... 66

Gloucester fish and potash ................ 66

Highly nitrogenized mixture $\ldots . \ldots \ldots \ldots \ldots . . . .2$

Hill and drill phosphate ................... 66

Lawn and garden dressing $\ldots \ldots \ldots \ldots \ldots \ldots . . \ldots 6,66$

Market garden fertilizer ................. 59, 66

Potato and vegetable fertilizer ............ 66

phosphate $\ldots \ldots \ldots \ldots \ldots .68$

Special crop grower ..................... 2

Sure crop phosphate ...................... 68

Tobacco starter ....................... 68

Stockbridge Special complete manure for corn and all grain crops ............................

Special complete manure for potatoes and vegetables $\ldots \ldots \ldots \ldots \ldots \ldots \ldots \ldots \ldots$.

Special complete manure for seeding down, permanent dressing and legumes ...........

Special complete manure for tobacco ........

Special complete manure for top dressing and

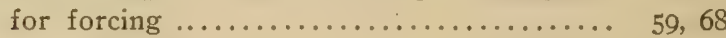

Bowker's Dry ground fish ....................... 55 
Bowker Fertilizer Co., cont'd:-

Page

Bowker's Fresh ground bone .................. 46

Market bone ....................... 46

Pure unleached Canada hardwood ashes ..... 93, 94

Tobacco ash elements ................... 68, 82

Acid phosphate ................... 40, 4 I

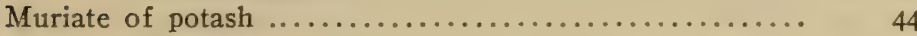

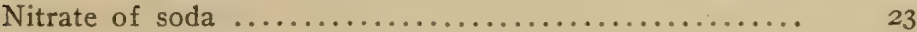

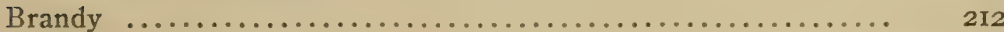

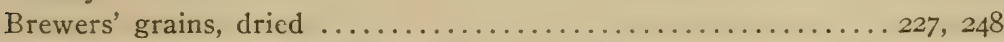

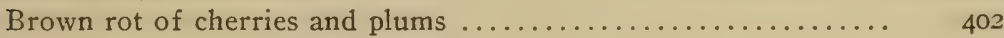

Browntail moth, checking the spread of the $\ldots \ldots \ldots \ldots \ldots \ldots$ 28I

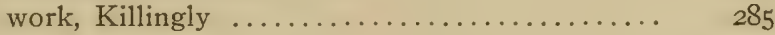

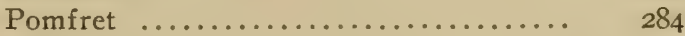

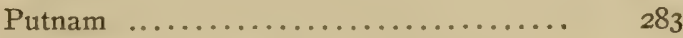

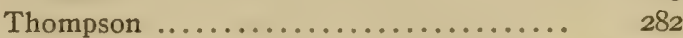

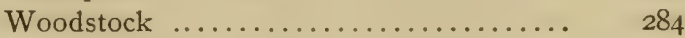

Buckwheat products ................................ 248

Buffalo Fertilizer Co.:-

Buffalo Celery and potato special ................. 68

Farmers choice ....................... 58, 68

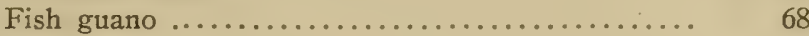

H. G. Manture ..................... 68,82

New England special .................... 68

Tobacco producer ....................... 68

Top dresser .......................... 60, 68

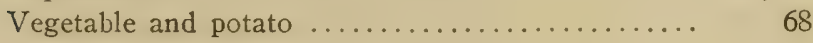

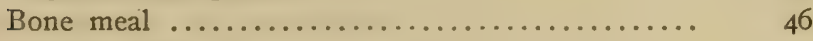

Ground bone ......................... 46

Lime-kiln ashes ...................... 9I

Tankage ............................ 49, 50

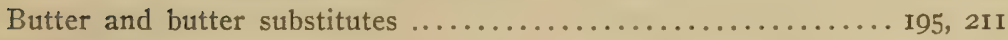

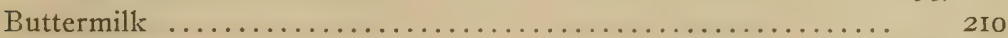

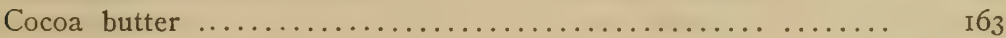

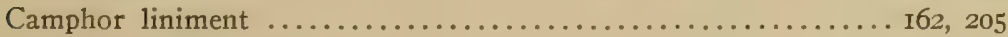

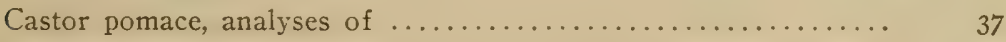

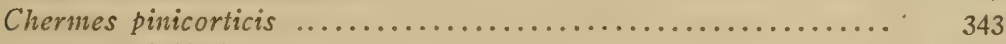

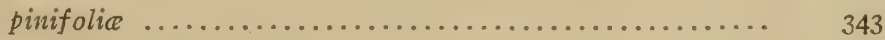

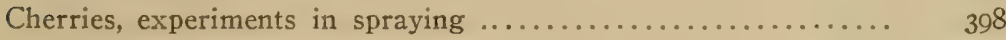

recommendations for spraying ............... 404

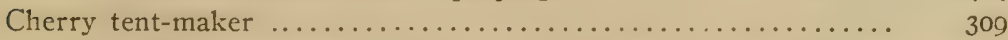

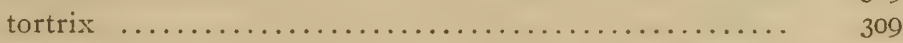

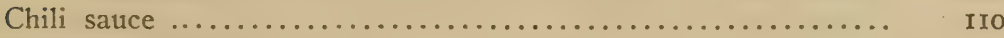

Chittenden, The E. D., Co.:-
Chittenden's Complete tobacco and onion grower ........ 68

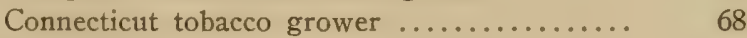

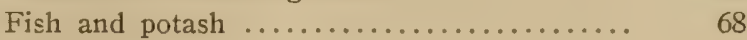


Chittenden, The E. D., Co., cont'd:-

Page

Chittenden's Potato and grain 70 manure

60,70

Tobacco special 70

Chocolate methods of analysis of IO2 milk sweet I08 104

Chop feeds 228,250

Chrysanthemum leaf-miner 342

Chrysopa 289

Cicada, appearance of the periodical ..................... $2 y 6$

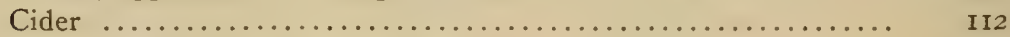

Clark Seed Co., The Everett B.:-

Clark's Special mixture for general use ........... 58,70

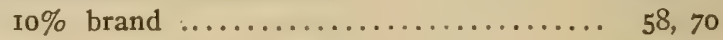

Clisiocampa americana .......................... 342

Cob meal ......................................... 246

Cocoa .......................................... I95

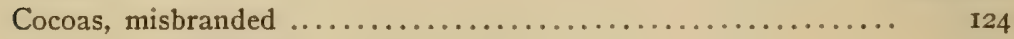

Cod liver oil, methods of analysis of $\ldots \ldots \ldots \ldots \ldots \ldots \ldots \ldots$ I70

wine of the extract of $\ldots \ldots \ldots \ldots \ldots \ldots \ldots \ldots$ I7I

emulsions ........................ 163, 205

Coe-Mortimer Co.:-

E. Frank Coe's Celebrated special potato fertilizer ...... 60, 70

Complete manure with 10\% potash ..... 70

Gold brand excelsior guano ..........58, 60, 70

H. G. Ammoniated bone superphosphate .. 70

New Englander corn and potato fertilizer 60,70

Red brand excelsior guano .......... 60,70

Tobacco and onion special ........... 70

Peruvian Tobacco fertilizer .................. 70, 82

Vegetable grower ................ 60, 70

XXX Pure ground bone .................... 46

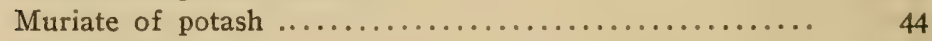

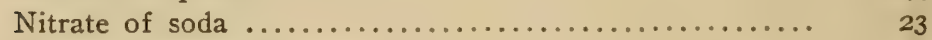

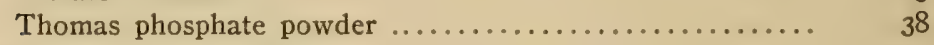

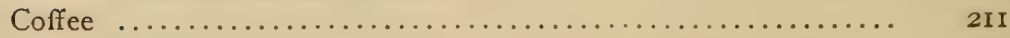

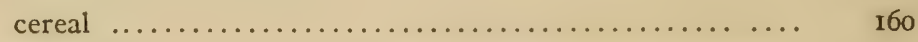

Condensed milk ............................... 200

Connecticut Valley Orchard Co.:-

C. V. O. Co.'s H. G. complete fertilizer ........... 70, 84

Cooper's Fertilizer, Peter:-

Peter Cooper's Pure bone dust ................. 46

Copidosoma truncatella ......................... 328

Corn, see Maize.

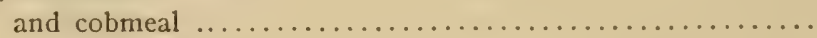


Corn and oat feeds ................................ 228, 250

breeding, dominance of characters in ................ 4 I

crosses between races differing in height of plants ...... 4 19

having different row number ..... 4 I 9

of flint and dent varieties .................. $4 \mathrm{I} 8$

or dent with sweet varieties of $\ldots \ldots \ldots \ldots$ 4II

purple and white varieties of $\ldots \ldots \ldots \ldots \ldots .4 . \ldots .4 \mathrm{I} 3$

yellow and white varieties of ............. $4 \mathrm{I} 2$

having red cob, crosses with varieties having white cob ... 415

pericarp, crosses with varieties having white

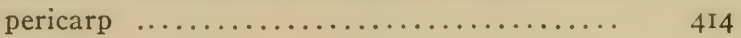

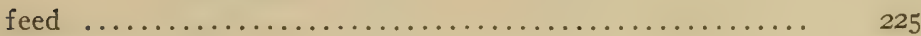

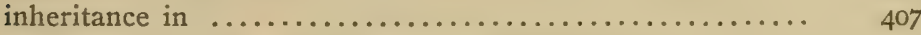

of crosses not showing dominance $\ldots \ldots \ldots \ldots . \quad 4 \mathrm{I} 8$

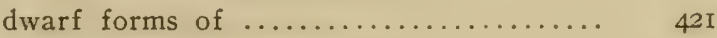

divided ears $\ldots \ldots \ldots \ldots \ldots \ldots \ldots \ldots \ldots \ldots, 422$

irregularity of rows of $\ldots \ldots \ldots \ldots \ldots \ldots .423$

length of ears and size of seeds ......... 420

summary of crosses showing dominance ............. 416

Cotton hull ashes, analyses of ....................... 92

moth ....................................267, 339

seed feed meal ............................... 221, 238

meal ................................ 220, 238

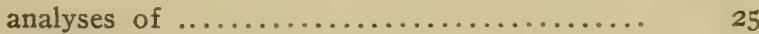

Cracker waste .................................... ${ }_{232}$

Cream .......................................... 195, 210

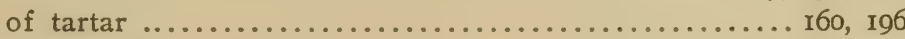

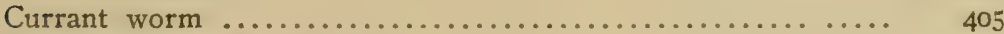

Currants, anthracnose of .......................... 405

the spraying of $\ldots \ldots \ldots \ldots \ldots \ldots \ldots \ldots \ldots \ldots \ldots, 405$

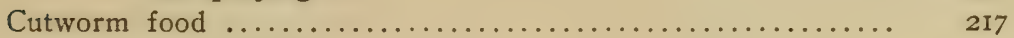

Cylindrosporium Podi ............................... 398

Cyllene robinic ................................... 345

Dairy feeds ................................. 229, 252

Diabetic foods. See gluten preparations.

Digestibility of feeds ............................. 233

Digestible nutrients purchasable for one dollar ............. 234

Dioryctria abietella ................................. 34

Dissolved rock phosphate, analyses of ................... 39

Distillers' grains, dried ...............................227, 250

Dominance of characters ............................ 409

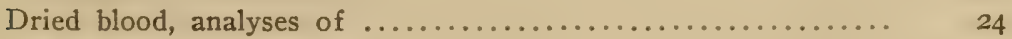

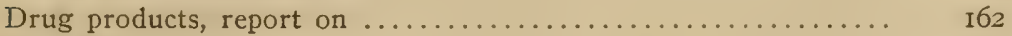

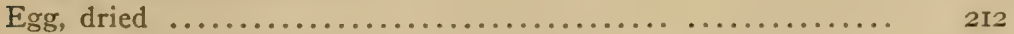

Eldredge, T. H. :-

Eldredge's Special fish and potash fertilizer ........... 70

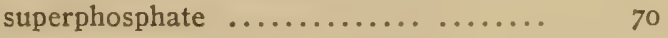


Elm scale ................................... 344

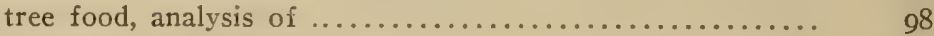

Entomosporium maculatum ...................... 396

Essex Fertilizer Co.:-

Essex Complete manure for corn, grain and grass ....... 70 potatoes, roots and vegetables 60,72

Fertilizer for grass and top dressing ........... 72

Market garden and potato manure ............. 72

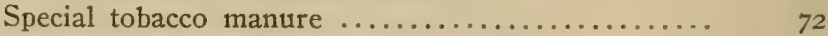

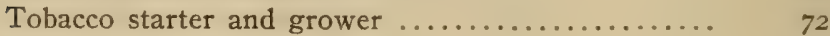

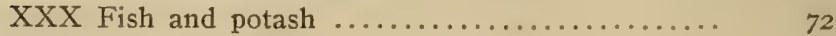

Ground bone ............................. 46

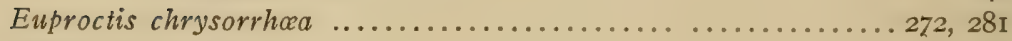

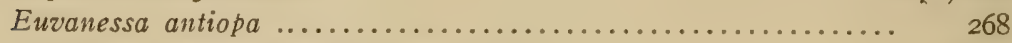

Exhibits of entomological department ................. 264

Expenditures of entomological department ............... 259

Feeding stuffs, abstract of law concerning ............... 2 I9

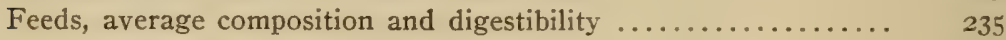

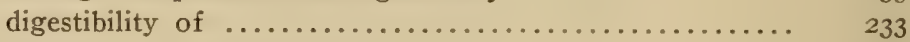

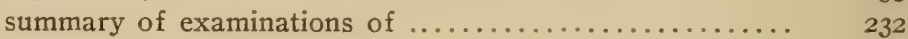

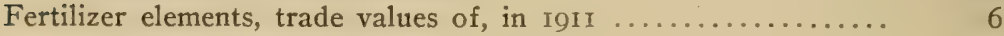

Law, summary of ........................ I

Fertilizer Materials Supply Co:-

Acid phosphate ......................... 40, $4 \mathrm{I}$

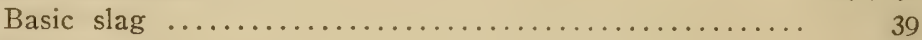

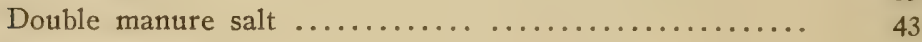

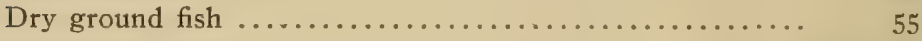

Muriate of potash ..................... 42, 44

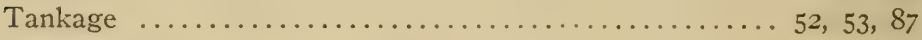

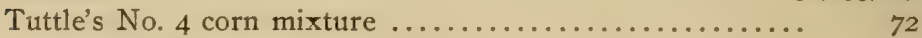

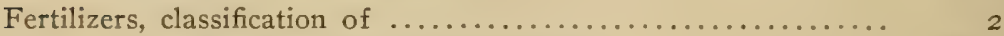

explanations concerning analyses .............. 3

mixed, method of valuation of $\ldots \ldots \ldots \ldots \ldots \ldots \ldots$.

number entered for sale .................... I

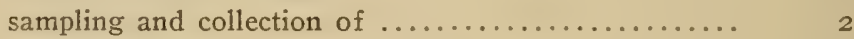

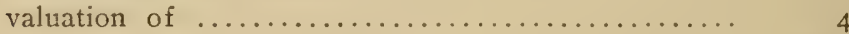

Fish ........................................... 2 II

analyses of dry ground and acidulated ............. 54

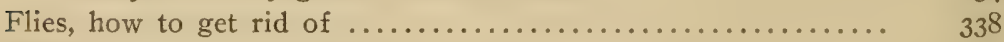

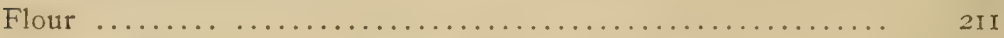

Food and drug products, summary of examinations of ...... 2 I5

products, report on ............................. IOI

Forest tent caterpillar ........................ 343

Frisbie, L. T., Co. :-

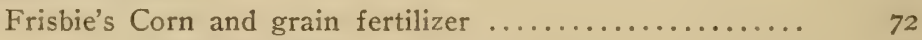

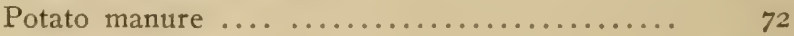

Vegetable grower $\ldots \ldots \ldots \ldots \ldots \ldots \ldots \ldots \ldots \ldots, 60,72$ 
Frisbie, L. T., Co., cont'd:-

Page

Frisbie's Fine bone meal ...................... $\quad 46$

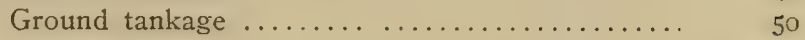

Acid phosphate .......................... 4I

Muriate of potash ...................... 44, 45

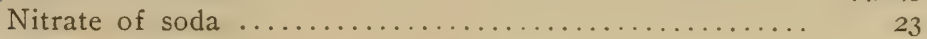

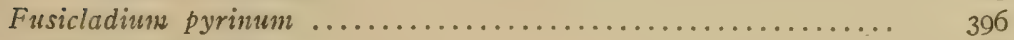

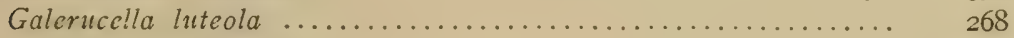

German Kali Works:-

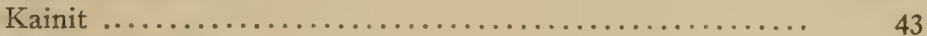

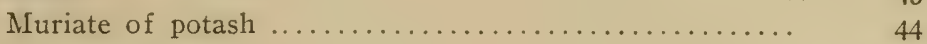

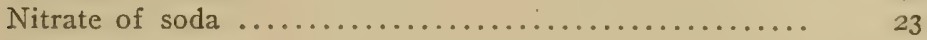

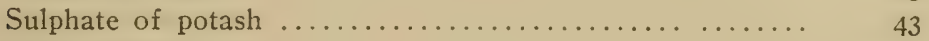

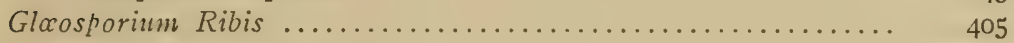

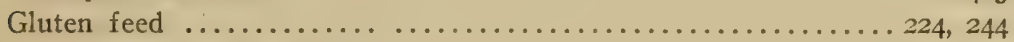

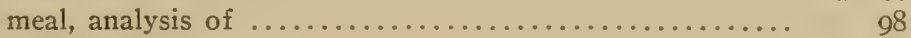

preparations ............................... 196

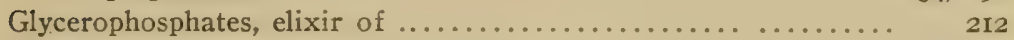

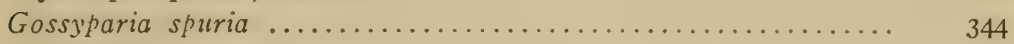

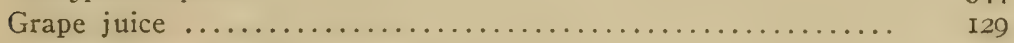

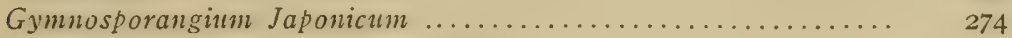

Gypsy moth, progress in controlling ................. 277

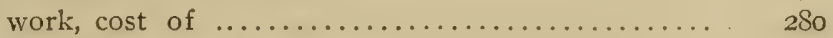

Stonington infestation .............. 278

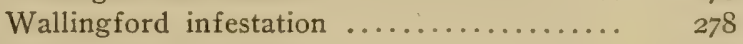

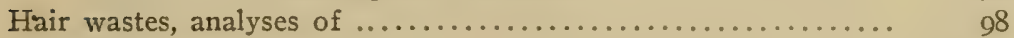

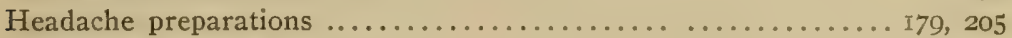

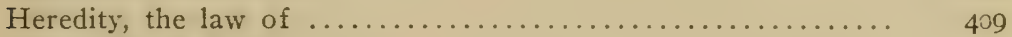

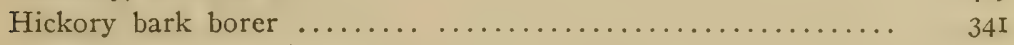

Home mixtures, analyses of $\ldots \ldots \ldots \ldots \ldots \ldots \ldots \ldots \ldots \ldots \ldots \ldots \ldots \ldots \ldots$

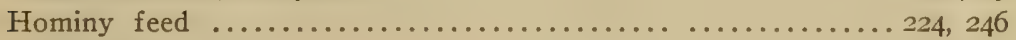

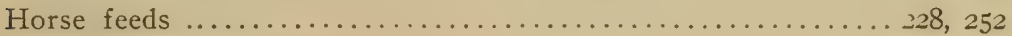

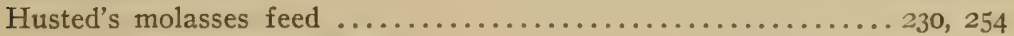

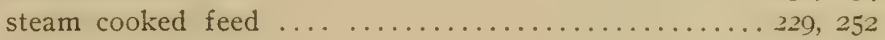

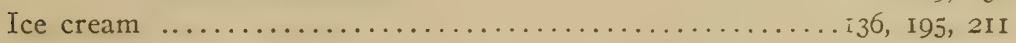

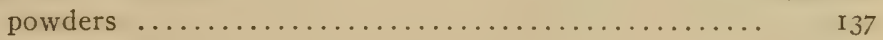

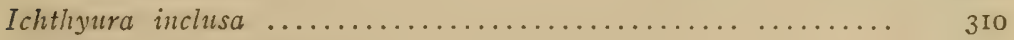

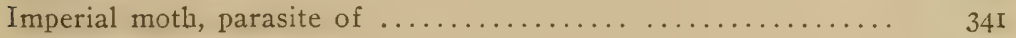

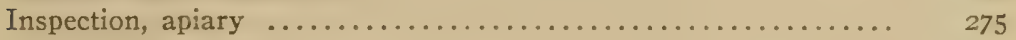

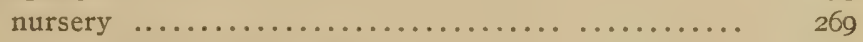

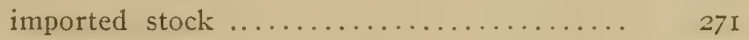

work, summary of entomological ............. 264

Iodine, tincture of $\ldots \ldots \ldots \ldots \ldots \ldots \ldots \ldots \ldots \ldots \ldots \ldots \ldots \ldots \ldots \ldots$ ISo

James, Ernest L.:-

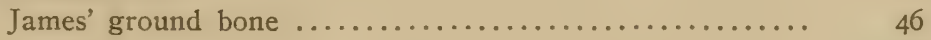

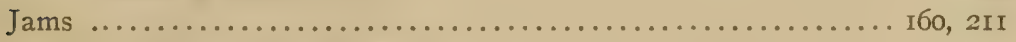

Juniperus chinensis var. compacta ................. 274 
Junipcrus tachikyaku

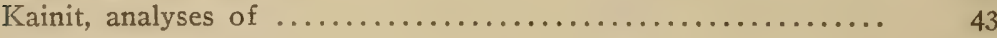

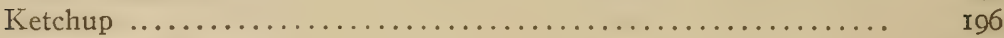

Lathromeris cicade .............................. 303

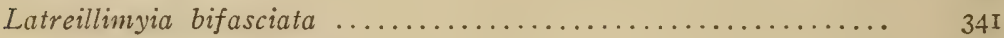

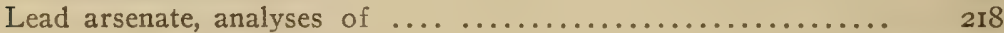

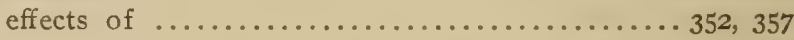

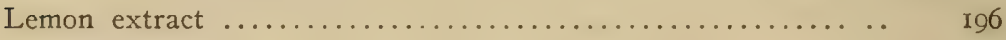

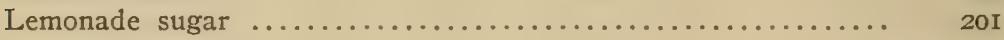

Leopard moth ............................. 3 I7

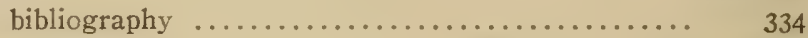

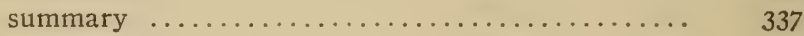

Leptinotarsa decemlineata .......................... 3 II

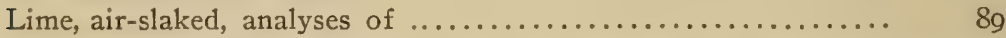
and sulphur, self-boiled ...353, 36r, 362, 373, 375, 400, 401, 402, 405 in various forms, analyses of $\ldots \ldots \ldots \ldots \ldots \ldots \ldots \ldots .8 .6 .60$

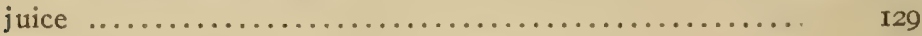

water $\ldots \ldots \ldots \ldots \ldots \ldots \ldots \ldots \ldots \ldots \ldots \ldots \ldots \ldots \ldots \ldots \ldots \ldots \ldots \ldots \ldots$

Lime-kiln ashes, analyses of $\ldots \ldots \ldots \ldots \ldots \ldots \ldots \ldots \ldots \ldots \ldots \ldots \ldots \ldots \ldots \ldots$ gr

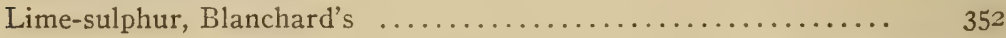

Grasselli's ........................ 362, 397

Niagara ........................... 353

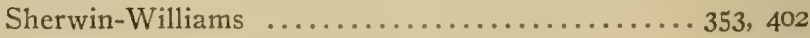

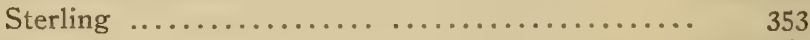

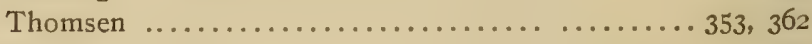

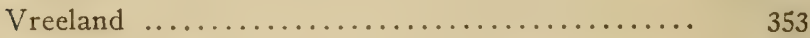

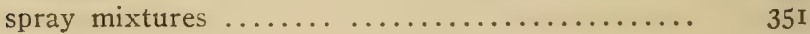

Limestone, analyses of $\ldots \ldots \ldots \ldots \ldots \ldots \ldots \ldots \ldots \ldots \ldots . \ldots \ldots \ldots$

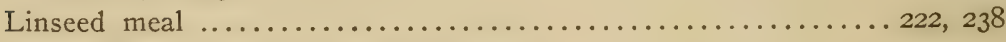

Listers Agricultural Chemical Works:-

Lister's Ammoniated dissolved bone phosphate ......... 72

Complete tobacco manure ................. 72

(carb.) $\quad \ldots \ldots \ldots \ldots \ldots \ldots, \quad 72$

Corn and potato fertilizer ................ 72

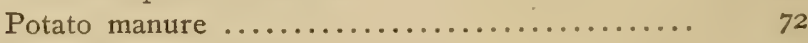

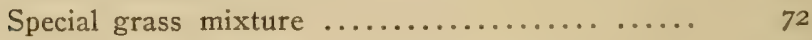

10 $\%$ fertilizer .................. 72

Standard pure bone superphosphate of lime $\ldots . .72$

Success fertilizer ................... 72

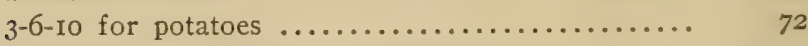

Celebrated ground bone and tankage ........... 50

Litomastix (Copidosoma) truncatella ................. 328

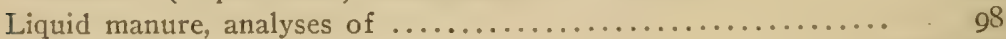

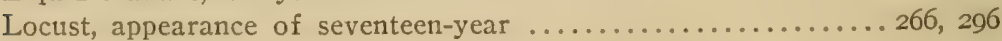

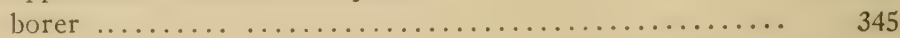

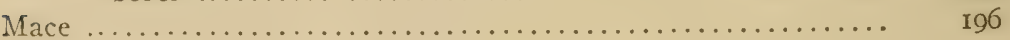


Page

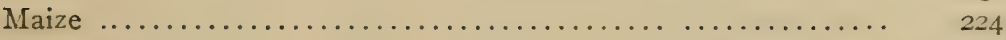

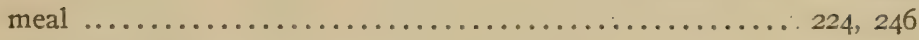

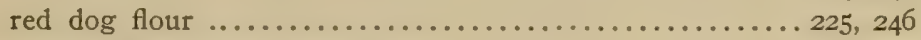

Malacosoma (Clisiocampa) anericana ................ 342

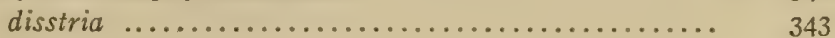

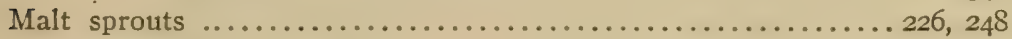

Manchester, E., \& Sons :-

Manchester's Formula .................. 72, 84

Mapes F. \& P. G. Co. :-

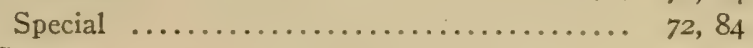

Average soil complete manure $\ldots \ldots \ldots \ldots \ldots \ldots \ldots \ldots .58,74$

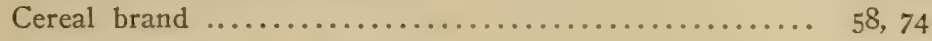

Complete manure " $\mathrm{A}$ " brand $\ldots \ldots \ldots \ldots \ldots \ldots \ldots \ldots \ldots$ 58, 74

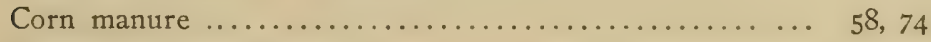

Economical potato manure .................. 58,74

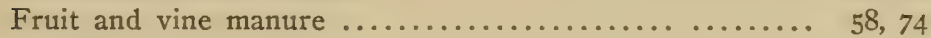

Potato manure .......................... 58, 74

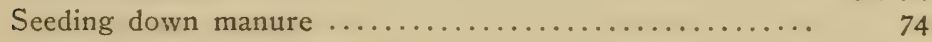

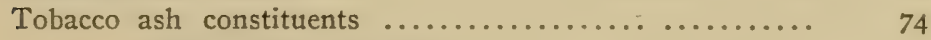

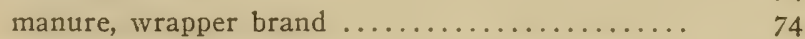

starter, improved ................... 58, 74

Top dresser improved, full strength .............. 74

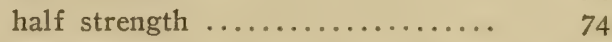

Vegetable or complete manure for light soils ........ 58, 74

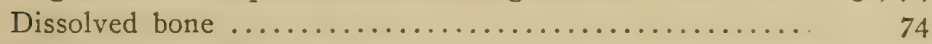

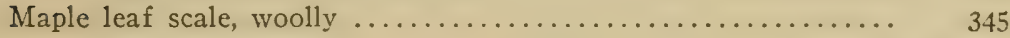

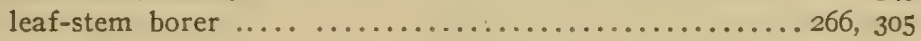

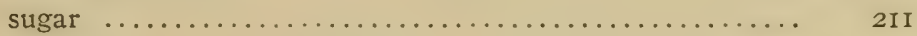

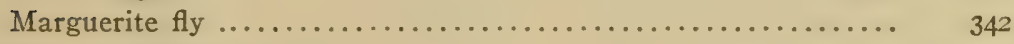

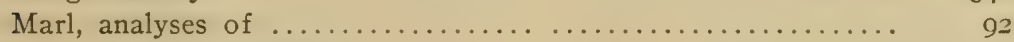

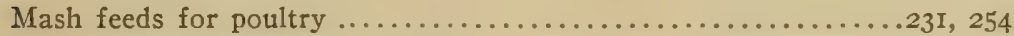

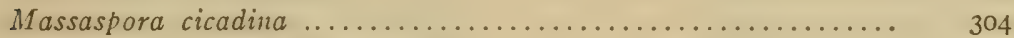

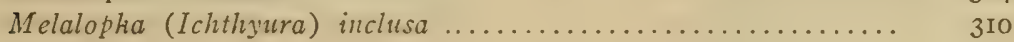

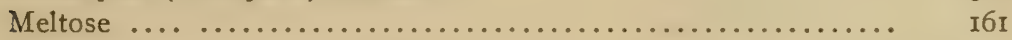

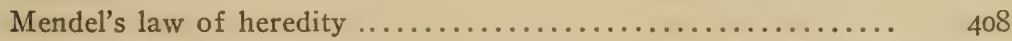

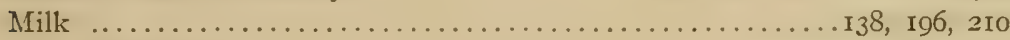

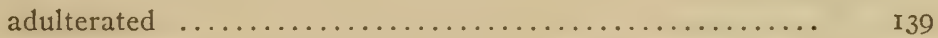

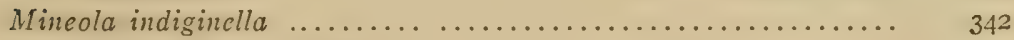

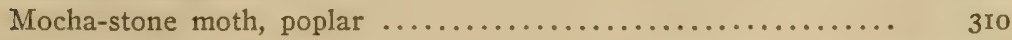

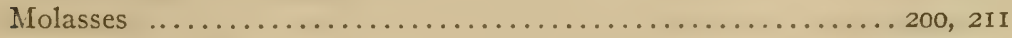

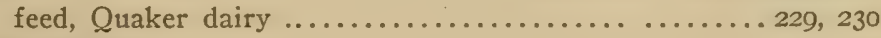

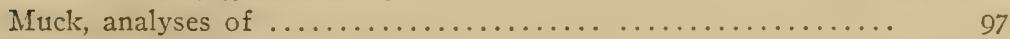

National Fertilizer Co. :-

National Ammoniated bone phosphate .............. 74

Complete corn and grain fertilizer ........... 74

grass fertilizer................. $\quad 74$ 
National Fertilizer Co., cont'd:-

National Complete root fertilizer

tobacco fertilizer .......................

Page

\section{National Complete root fertilizer.}

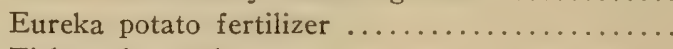

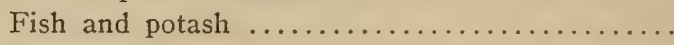

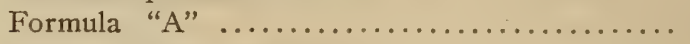

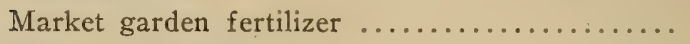

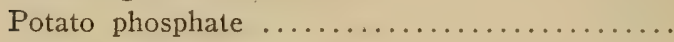

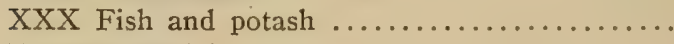

Tobacco special

(carb.)

Soluble bone and potash $\ldots \ldots \ldots \ldots \ldots \ldots \ldots$

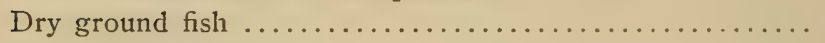

60,74

74

Natural Guano Co. :-

Sheep's head brand of pulverized sheep manure ........ 46,48

New England Fertilizer Co.:-

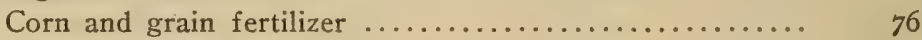

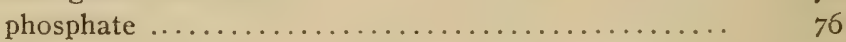

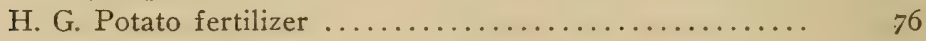

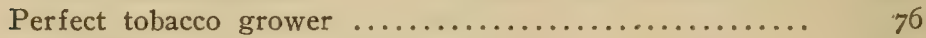

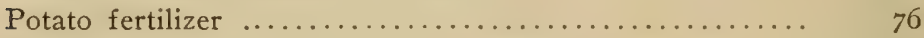

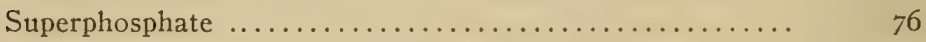

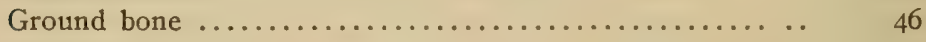

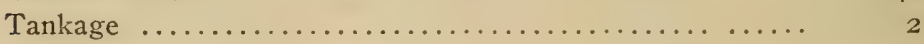

Niantic Menhaden Oil \& Guano Co.:-

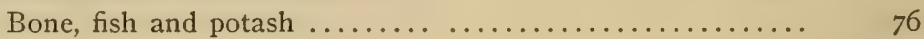

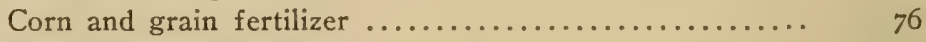

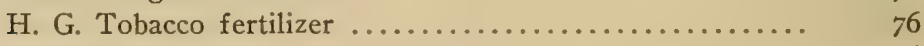

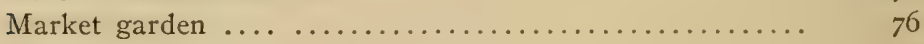

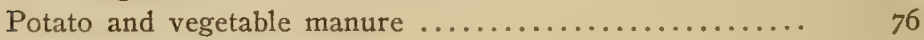

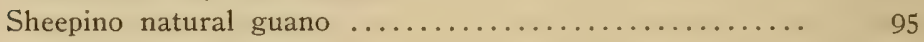

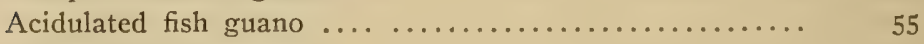

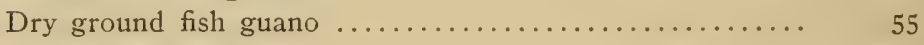

Nitrate Agencies Co.:-

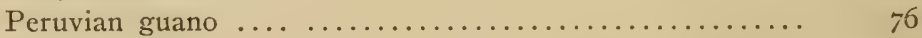

Acid phosphate ....................... 40, 4I

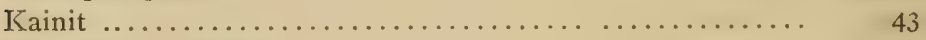

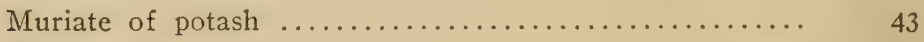

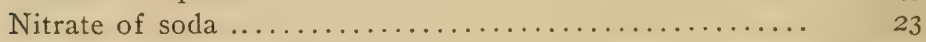

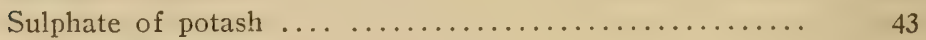

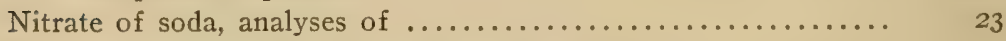

Nitrogen availability, pot experiments on ............... I4

in mixed fertilizers, solubility of organic ......... 56

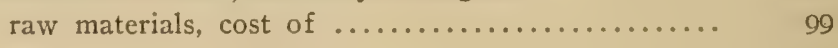


Nitrogen "inactive"

modified neutral permanganate method to determine

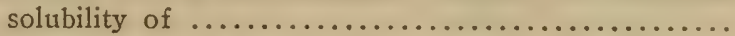

of raw materials, solubility of organic by the alkaline

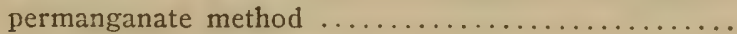

solubility of organic $\ldots \ldots \ldots \ldots \ldots \ldots \ldots \ldots \ldots \ldots$

Nitrogenous superphosphates, guaranties of ................. 60

valuation of $\ldots \ldots \ldots \ldots \ldots \ldots .6 \%$

and guanos, analyses of $\ldots \ldots \ldots .62-85$

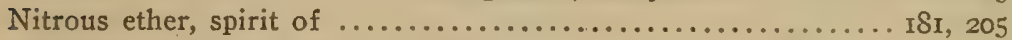

Nurseries, inspection of Connecticut ................... $\quad 269$

Nursery firms receiving certificates in I9II, list of .......... 270

stock, inspection of imported ................. 271

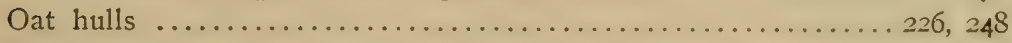

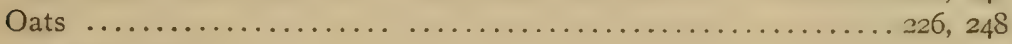

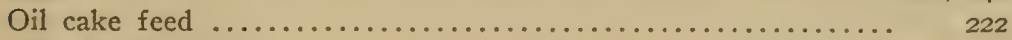

Olds \& Whipple:-

O. \& W.'s Complete tobacco fertilizer ............... $\quad 76$

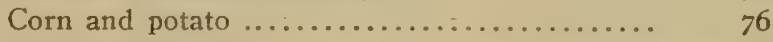

Fish and potash $\ldots \ldots \ldots \ldots \ldots \ldots \ldots \ldots \ldots \ldots . \ldots \ldots$

Grass fertilizer ...................... $\quad 76$

H. G. Potato fertilizer ................. 760

Special phosphate .................... $\quad 76$

Dry ground fish $\ldots \ldots \ldots \ldots \ldots \ldots \ldots \ldots \ldots \ldots$

Grey pomace $\ldots \ldots \ldots \ldots \ldots \ldots \ldots \ldots \ldots \ldots \ldots \ldots \ldots \ldots$

Vegetable potash ..................... $\quad 46$

Acid phosphate ...................... 40, 4I

Carbonate of potash $\ldots \ldots \ldots \ldots \ldots \ldots \ldots \ldots . . . \ldots \ldots$

Cotton hull ashes ..................... 92

H. G. Sulphate of potash ............... 43

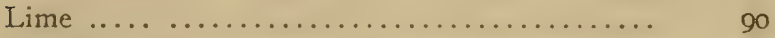

Precipitated bone ..................... 39

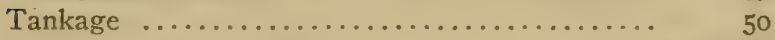

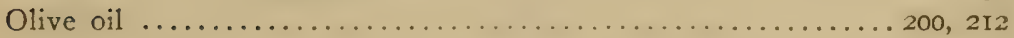

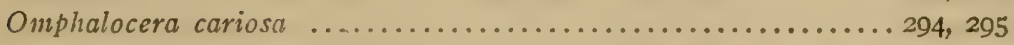

dentosa ................260, 263, 267, 292, 294, 295

Onion maggot ................................. ${ }_{28} 86$

Opium ...................................... 205

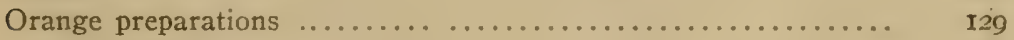

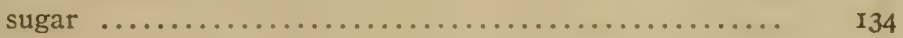

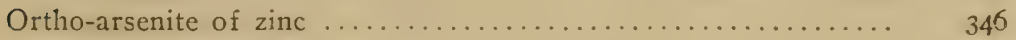

Pamphilius persicum ............................ $3 \mathrm{II}$

Paprika ...................................... ${ }_{\mathbf{I} 46}$

methods of analysis of ...................... $\quad 148$

Parmenter \& Polsey Fertilizer Co.:-

P. \& P. Grain grower ....................... 78 
Parmenter \& Polsey Fertilizer Co., cont'd:-

P. \& P. Plymouth Rock brand fertilizer ............ 78

Potato fertilizer ................... 78

grower .......................... $\quad 78$

Special tobacco grower ................. 78

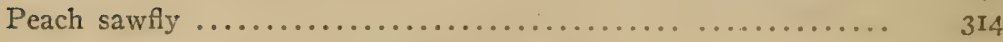

Ground bone ........................ 46

Peaches, benefits of spraying ..................... 374

details of spraying experiments ................... $39 \mathrm{I}$

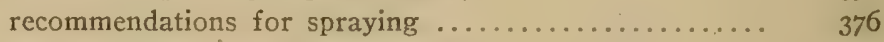

results of two years' experiments .................. 37 I

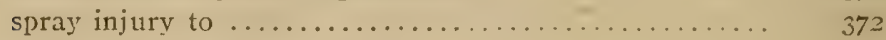

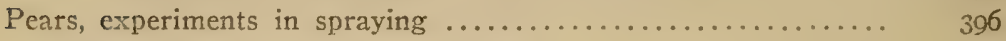

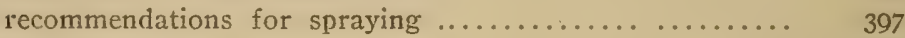

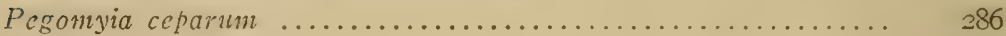

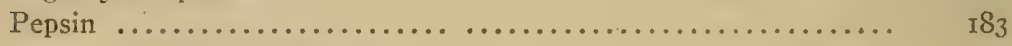

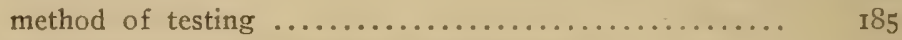

Percentage difference, meaning and calculation of .......... 84

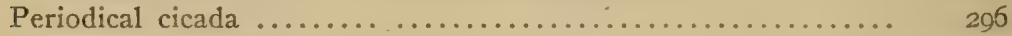

Phenacoccus acericola ................................ 345

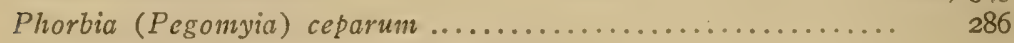

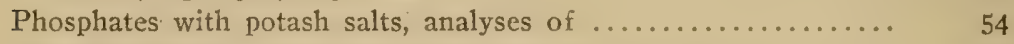

Phosphoric acid in raw materials, cost of ............... I00

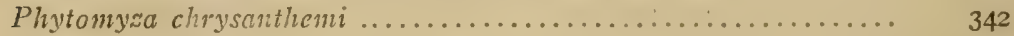

Pine aphids, woolly ............................... 343

Pissodes strobi ..................................... 268, 307

Plowrightia morbosa .......................... 399

Plums, experiments in spraying ..................... 398

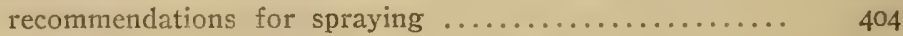

Plusia brassice ................................ 328

Poplar mocha-stone moth or tent-maker ............... 310

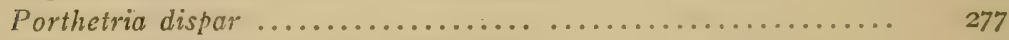

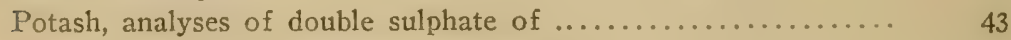

high grade sulphate of ............. 43

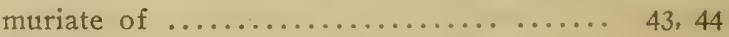

analysis of carbonate of $\ldots \ldots \ldots \ldots \ldots \ldots \ldots \ldots \ldots \ldots \ldots \ldots \ldots \ldots$

misbranded muriate of $\ldots \ldots \ldots \ldots \ldots \ldots \ldots, \quad 45$

in raw materials, cost of .................... 100

Potassium iodide, determination of, in extract of sarsaparilla ... 193

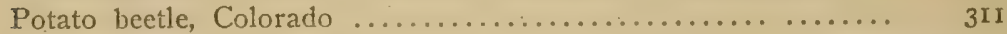

Poultry feeds ........................................ 254

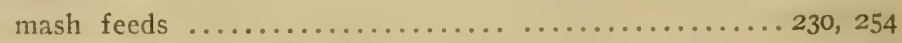

Precipitated bone phosphate, analyses of .............. 39

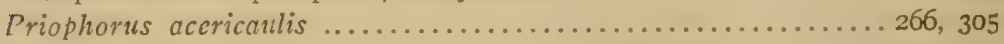

Protena dairy feed ...................................... 254

Pteronus ribesi ................................ 405 
Pulverized Manure Co.:-

Page

Wizard brand manure......................... 95

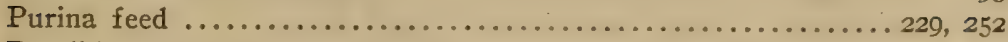

Pyralid ................................... 292

Quinces, experiments in spraying .................... 396

Quinine pills

recommendations for spraying ................. 398

Raspberry syrup ................................ 129, 106

Recessive characters ............................. 409

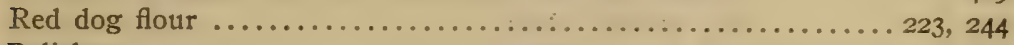

Relishes .....................................

Rhagoletis cingulata ............................. $\quad 399$

Røestelia aurantiaca .............................. $\quad 396$

Rogers \& Hubbard Co.:-

Hubbard's "Bone Base" Complete phosphate ........... 78

Grass and grain or fruit fertilizer $\quad 78$

New market garden phosphate ... $\quad 78$

Oats and top dressing ......... 78

Potato phosphate ............. 60, 78

Soluble corn and general crops manure .................. 78

Soluble potato manure .......... 78

tobacco manure ......... $\quad 78$

Pure raw knuckle bone flour ..... , 46

Rogers Mfg. Co.:-

Strictly pure fine bone .......... 46

All round fertilizer .......................... $\quad 78$

Complete corn and onion ..................... 78

pctato and vegetable .................... 78

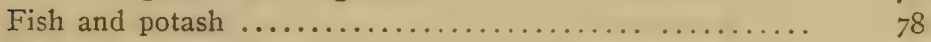

H. G. Grass and grain ........................ 78

Oats and top dressing ...................... $\quad 78$

Soluble tobacco ......................... $\quad 78$

Tobacco and potato ....................... 78

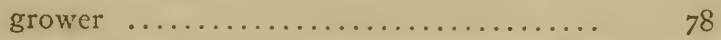

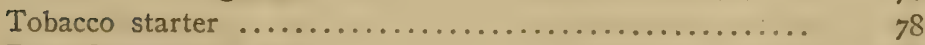

Pure fine ground bone ....................... 46

knuckle bone flour ........................ ; 46

Acid phosphate .......................... 40, 4I

H. G. Sulphate of potash ...................... 43

Muriate of potash ............................. 43

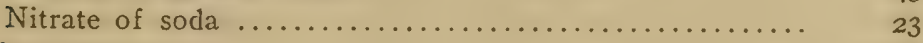

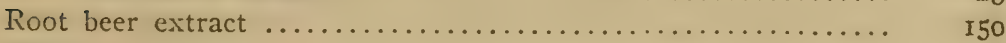

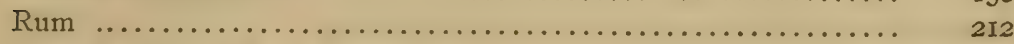

Rust on juniper from Japan ....................... 274

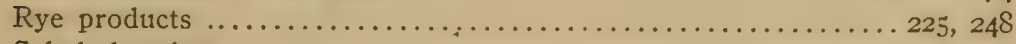

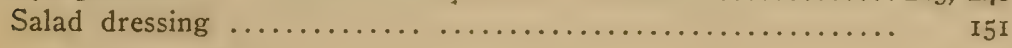


Sanderson Fertilizer \& Chemical Co.:-

Page

Kelsey's Bone fish and potash

80,84

Sanderson's Atlantic coast bone fish and potash

Corn superphosphate

Formula A

B

Potato manure

Special with 10\% potash

Top dressing for grass and grain

Fine ground bòne 46,48

fish

Muriate of potash

43,44

Nitrate of soda

Thomas slag

Special mixture $4-8-8 \ldots \ldots \ldots \ldots \ldots \ldots \ldots . . . \ldots \ldots$

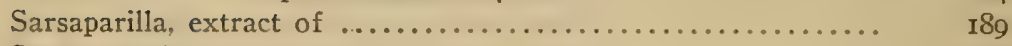

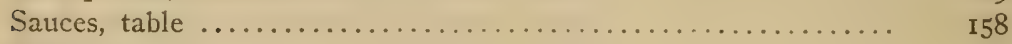

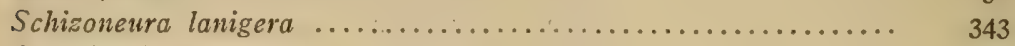

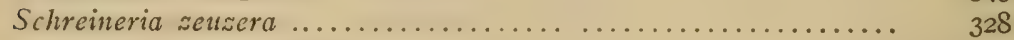

Schumacher's calf meal ......................... 230, 254

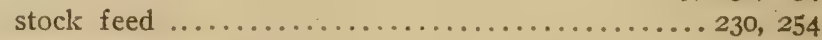

Scolytus quadrispinostus ................................ $34 \mathrm{I}$

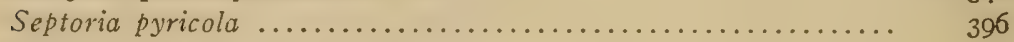

Seventeen-year locust in Connecticut in Igr . ............. 296

Shay, The C. M., Fertilizer Co.:-

Shay's Corn fertilizer ...................... 80

Grass fertilizer ..................... 8o

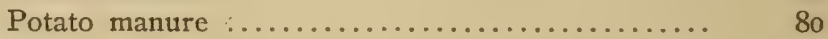

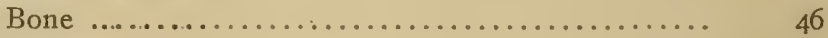

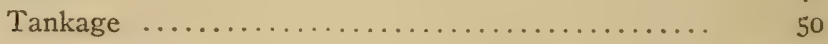

Acid phosphate .................... 40, 4I

Chrysanthemum fertilizer ................. 98

Muriate of potash ................. 44, 45

Nitrate of soda ..................... 23

Special mixture ................... 80, 84

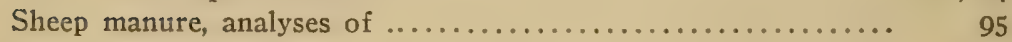

Shoddy waste, analyses of $\ldots \ldots \ldots \ldots \ldots \ldots \ldots \ldots \ldots \ldots \ldots \ldots \ldots \ldots$

Shoemaker, M. L., \& Co.:-

"Swift-Sure" Superphosphate for general use .......... 80

potatoes ............. 80

truck, corn and onions .. 80

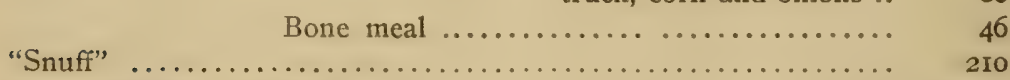

Soda, analyses of nitrate of $\ldots \ldots \ldots \ldots \ldots \ldots \ldots \ldots \ldots \ldots \ldots . \ldots \ldots . \ldots \ldots$

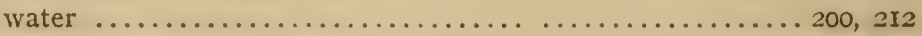

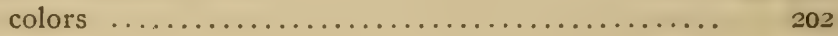


Soda water flavors

Page

syrups

202

200

Soups, condensed

I6r

Sphecius speciosus

Spray mixtures, cost of ......................... 349

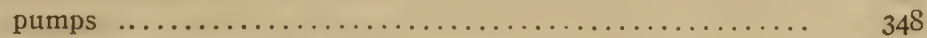

Sprayers, gasoline power ........................ 348

Sprays on apples, peaches, etc., tests of summer ............ 347

Steero Bouillon Cubes ............................ I62

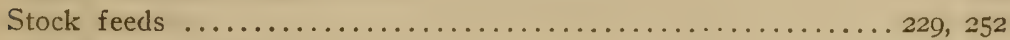

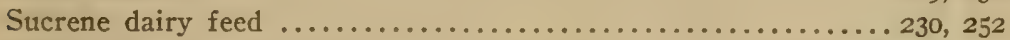

Sulphur and lead arsenate ......................357, 362, 367, 369

sprays, Bogart's sulphur compound .............. 355, 362

One-for-all $\ldots \ldots \ldots \ldots \ldots \ldots \ldots \ldots \ldots \ldots \ldots \ldots \ldots, 362$

Sulfocide $\ldots \ldots \ldots \ldots \ldots \ldots \ldots \ldots . . \ldots \ldots 5,362,369,373$

Swift's Lowell Fertilizer Co.:-

Swift's Lowell Animal brand ...................... 80

Bone fertilizer .................... 82

Empress brand $\ldots \ldots \ldots \ldots \ldots \ldots \ldots \ldots \ldots$. $\quad 82$

Potato manure .................... $8_{2}$

phosphate $\ldots \ldots \ldots \ldots \ldots \ldots \ldots . . .62$

Perfect tobacco grower $\ldots \ldots \ldots \ldots \ldots \ldots \ldots$ \&o

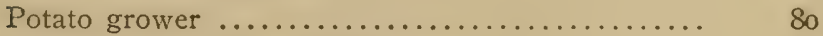

Special corn and vegetable manure...........$\quad 80$

grass mixture ..................... so

potato fertilizer .................... So

Superior fertilizer ...................... 80

Tobacco manure ......................... 80

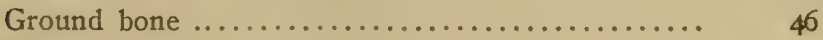

Nitrate of soda ............................. 2

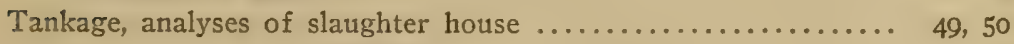

spurious $\ldots \ldots \ldots \ldots \ldots \ldots \ldots \ldots \ldots \ldots \ldots, \quad 52$

method of valuation $\ldots \ldots \ldots \ldots \ldots \ldots \ldots \ldots \ldots, \quad 7$

Tanner \& Wilcox:-

Tanner \& Wilcox' Reliable potato and garden phosphate .. 82

Tent caterpillar .................................. 342

Theobroma, oil of .................................. ${ }_{163}$

Thomas slag, analyses of ......................... ${ }_{3} 8$

Tibicen septendecin ................................ $\quad{ }_{296}$

Tmetocera ocellana .............................. 267

Tobacco ashes, analyses of .......................... 95

stems, analyses of .......................... 96

Totty's carnation fertilizer, analysis of .................... 98

Trade values of fertilizer elements in Igri ................ 6

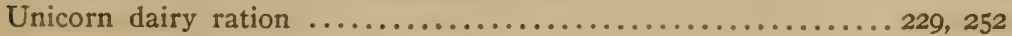

Union grains, Biles' ready ration ...................229, 252 
Valuation of bone and tankage, method of

Valuations, explanations concerning

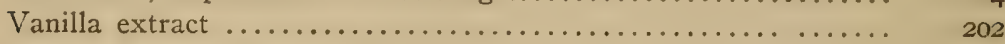

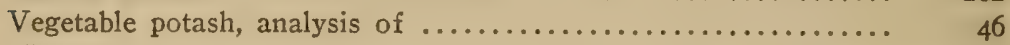

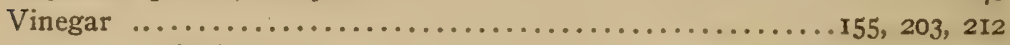

methods of analysis of $\ldots \ldots \ldots \ldots \ldots \ldots \ldots \ldots \ldots \ldots \ldots \ldots \ldots \ldots \ldots \ldots$

Weather conditions, effect on spraying ................ 363

Wells \& Downs :-

W. \& D. Special economical 6-8-5 .............. 82

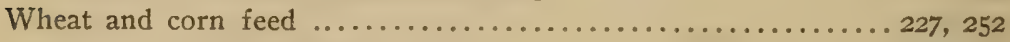

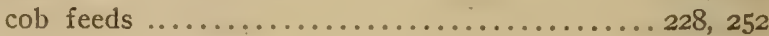

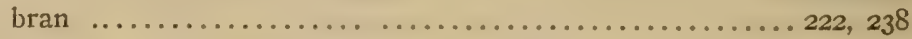

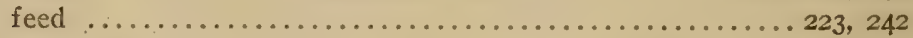

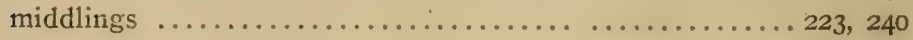

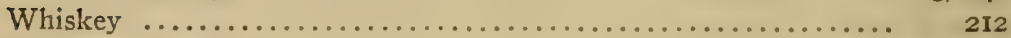

White pine weevil ................................ 268, 307

Wilcox Fertilizer Co. :-

Wilcox Complete bone superphosphate ............ 82

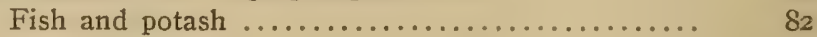

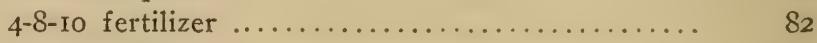

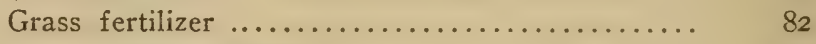

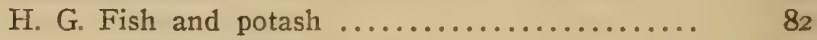

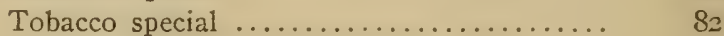

Potato fertilizer ..................... $8_{2}$

onion and vegetable phosphate ........ 82

Special superphosphate ................ 82

Dry ground acidulated fish ............ 54, 55

fish guano .................. 55

Ground steamed bone .................... 2

H. G. Tankage .................... 49. 50

Pure ground bone .................... 46

Acid phosphate .................... 40, 4I

Basic slag meal ....................... 38

H. G. Sulphate of potash ............... 43

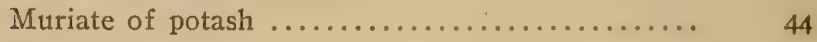

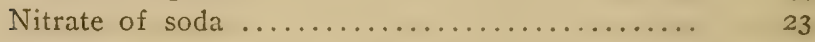

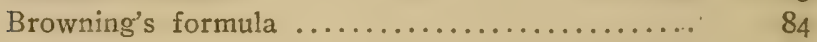

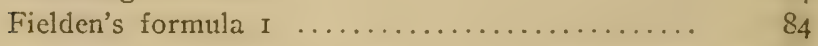

$2 \ldots \ldots \ldots \ldots \ldots \ldots \ldots \ldots \ldots \ldots \ldots \ldots \ldots$

I, grain and potatoes ........ 84

2 , grass $\ldots \ldots \ldots \ldots \ldots \ldots \ldots \ldots \quad 84$

Wine of the extract of cod liver oil ................... I7I

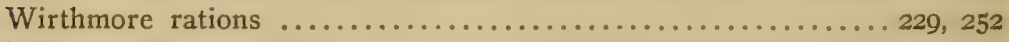

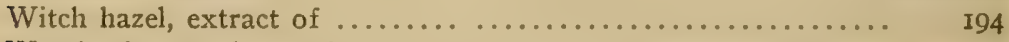

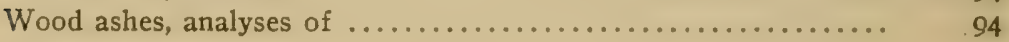


Page

Woodruff, S. D., \& Sons:-

Woodruff's Home mixture

58,82

Worcestershire and other sauces

Zeusera asculi

3 I 7

pyrina

$266,317,320$

Zinc, ortho-arsenite of

analysis of 



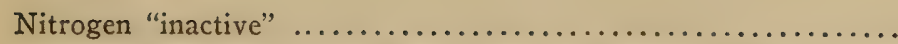

Page

modified neutral permanganate method to determine

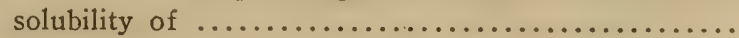

of raw materials, solubility of organic by the alkaline

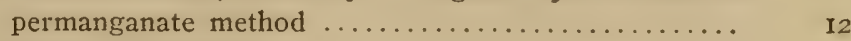

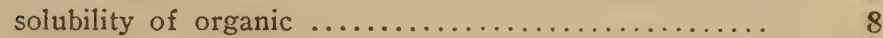

Nitrogenous superphosphates, guaranties of $\ldots \ldots \ldots \ldots \ldots \ldots .60$

valuation of $\ldots \ldots \ldots \ldots \ldots \ldots \ldots$. 6 I

and guanos, analyses of $\ldots \ldots \ldots .62-85$

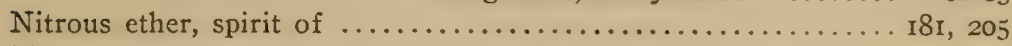

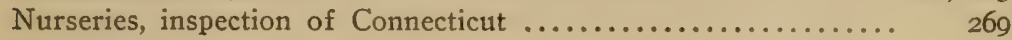

Nursery firms receiving certificates in IgI, list of .......... 270

stock, inspection of imported ................... $27 \mathrm{I}$

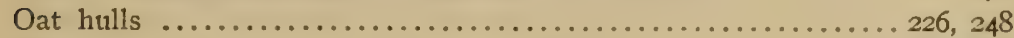

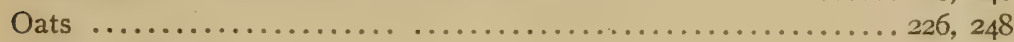

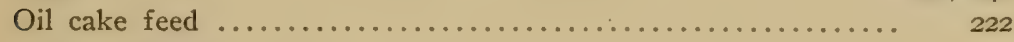

Olds \& Whipple:--

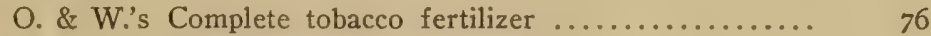

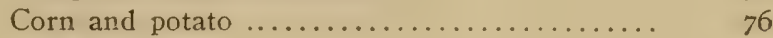

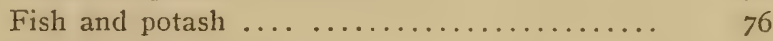

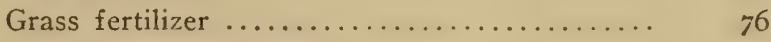

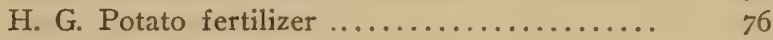

Special phosphate ....................... 76

Dry ground fish $\ldots \ldots \ldots \ldots \ldots \ldots \ldots \ldots \ldots \ldots \ldots$

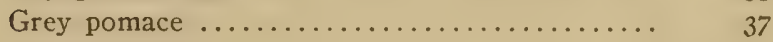

Vegetable potash ................... 46

Acid phosphate..................... 40, $4 \mathrm{I}$

Carbonate of potash ................ 45

Cotton hull ashes .................. 92

H. G. Sulphate of potash ............. 43

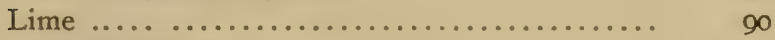

Precipitated bone ................. 39

Tankage .......................... 50

Olive oil ......................................... 2 r2

Omphalocera cariosa ..................................... 295

dentosa .................260, 263, 267, 292, 294, 295

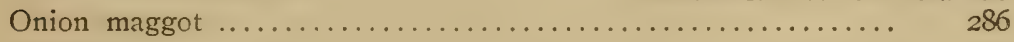

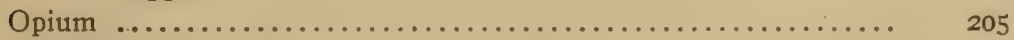

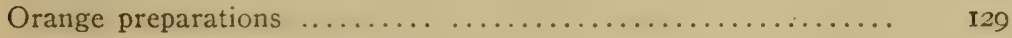

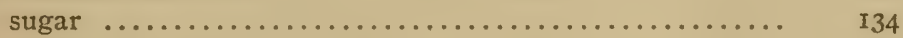

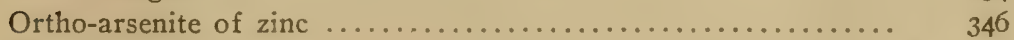

Pamplitius persicum ....................... 314

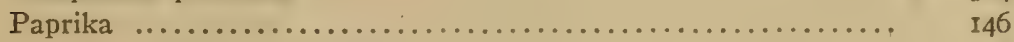

methods of analysis of .......................... 148

Parmenter \& Polsey Fertilizer Co.:-

P. \& P. Grain grower ....................... 78 
Parmenter \& Polsey Fertilizer Co., cont'd :-

Page

P. \& P. Plymouth Rock brand fertilizer ............. 78

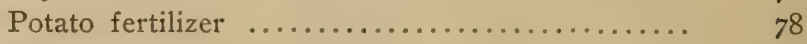

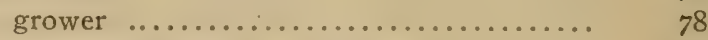

Special tobacco grower ................. 78

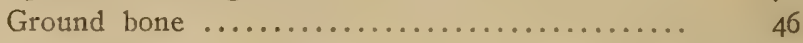

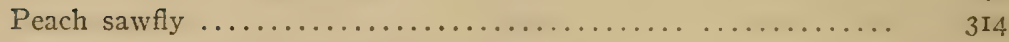

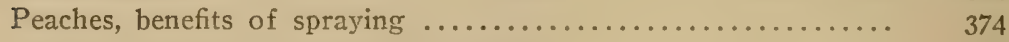

details of spraying experiments .................... $39 \mathrm{I}$

recommendations for spraying ............................... 376

results of two years' experiments ................. 37 I

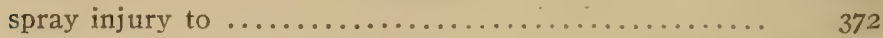

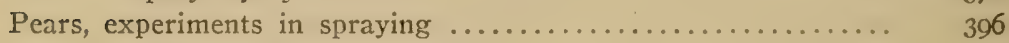

recommendations for spraying ............... 397

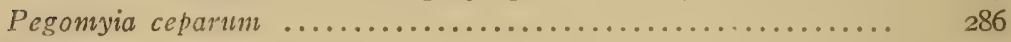

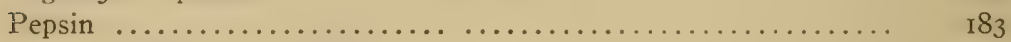

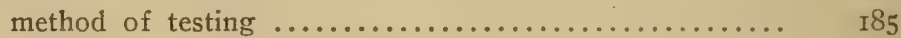

Percentage difference, meaning and calculation of ........... 84

Periodical cicada ................................ 296

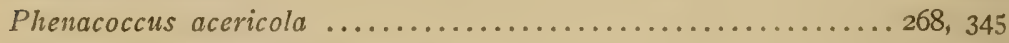

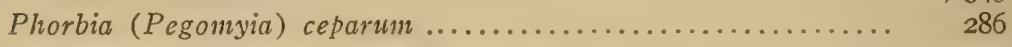

Phosphates with potash salts, analyses of .............. 54

Phosphoric acid in raw materials, cost of ................ I00

Phytomyza chrysanthemi ....................... 342

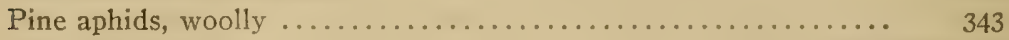

Pissodes strobi ............................ 63, 268, 307

Plowrightia morbosa ........................... 399

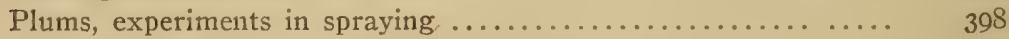

recommendations for spraying ............... 404

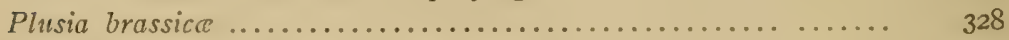

Poplar mocha-stone moth or tent-maker ................ 310

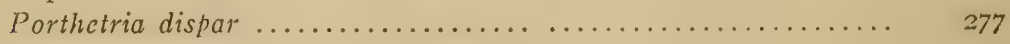

Potash, analyses of double sulphate of ................. 43

high grade sulphate of ............. 43

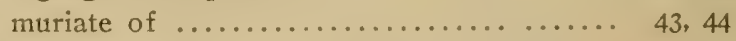

analysis of carbonate of $\ldots \ldots \ldots \ldots \ldots \ldots \ldots \ldots \ldots \ldots . \ldots \ldots$

misbranded muriate of $\ldots \ldots \ldots \ldots \ldots \ldots \ldots . . . \ldots$

in raw materials, cost of $\ldots \ldots \ldots \ldots \ldots \ldots \ldots \ldots \ldots \ldots$

Potassium iodide, determination of, in extract of sarsaparilla ... I93

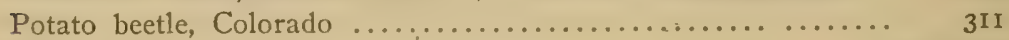

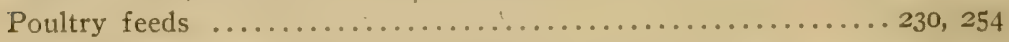

mash feeds ............................. 230, 254

Precipitated bone phosphate, analyses of ............... 39

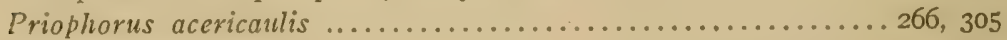

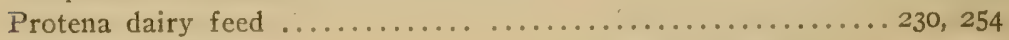

Ptcromus ribcsi ................................ 405 
Pulverized Manure Co.:-

Page

Wizard brand manure ......................... 95

Purina feed ................................ 229, 252

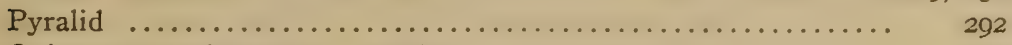

Quinces, experiments in spraying ................... 396 recommendations for spraying ................. 398

Raspberry syrup ............................. 129, I96

Recessive characters ............................ 409

Red dog flour ................................. 223, 244

Relishes .................................... I5I

Rhagoletis cingulata ............................. $\quad 399$

Restelia aurantiaca .............................. $\quad 396$

Rogers \& Hubbard Co.:-

Hubbard's "Bone Base" Complete phosphate ........... 78

Grass and grain or fruit fertilizer $\quad 78$

New market garden phosphate ... 78

Oats and top dressing ......... 78

Potato phosphate ............ 60, 78

Soluble corn and general crops manure .................. 78

Soluble potato manure ......... 78

tobacco manure ......... 78

Pure raw knuckle bone flour..... 46

Rogers Mfg. Co.:-

Strictly pure fine bone ......... 46

All round fertilizer ......................... 78

Complete corn and onion ....................... $\quad 78$

potato and vegetable.................... 78

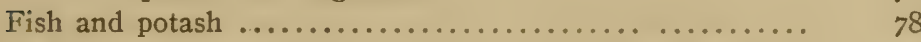

H. G. Grass and grain ......................... 78

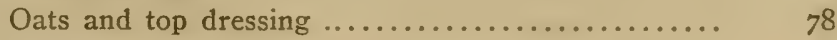

Soluble tobacco ......................... $\quad 78$

Tobacco and potato $\ldots \ldots \ldots \ldots \ldots \ldots \ldots \ldots \ldots, \quad 78$

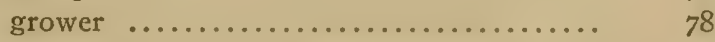

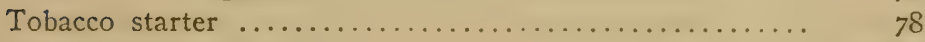

Pure fine ground bone ......................... $\quad 46$

knuckle bone flour ...................... $\quad 46$

Acid phosphate .......................... 40, 4I

H. G. Sulphate of potash ..................... 43

Muriate of potash ............................. 43

Nitrate of soda ............................. 23

Root beer extract ................................. ${ }_{150}$

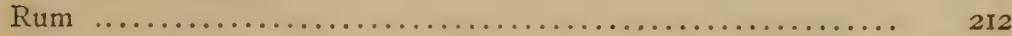

Rust on juniper from Japan ...................... 274

Rye products ...................................225, 248

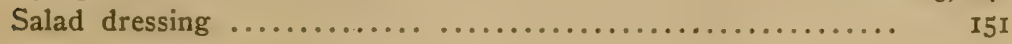




\section{Sanderson Fertilizer \& Chemical Co.:-}

Kelsey's Bone fish and potash

80,84

Sanderson's Atlantic coast bone fish and potash .........

Corn superphosphate

Formula A

B

Potato manure

Special with $10 \%$ potash ............... 80

Top dressing for grass and grain ........ 80

Fine ground bone $\ldots \ldots \ldots \ldots \ldots \ldots \ldots \ldots, 46,48$

fish $\ldots \ldots \ldots \ldots \ldots \ldots \ldots \ldots \ldots \ldots \ldots, \quad 55$

Muriate of potash ............... 43, 44

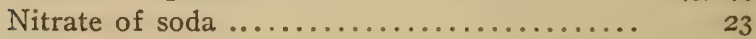

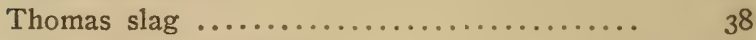

Special mixture $4-8-8 \ldots \ldots \ldots \ldots \ldots \ldots \ldots \ldots . . \ldots \ldots$

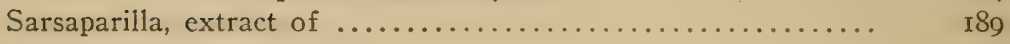

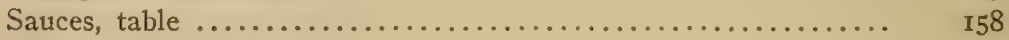

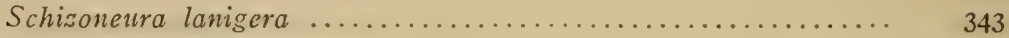

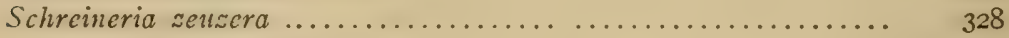

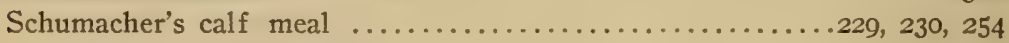

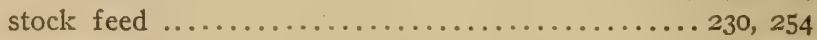

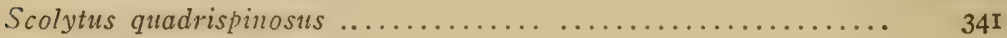

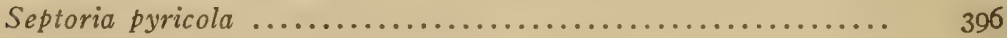

Seventeen-year locust in Connecticut in I9II .............. 296

Shay, The C. M., Fertilizer Co.:-

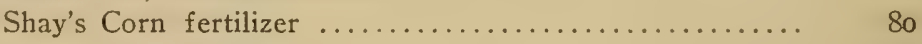

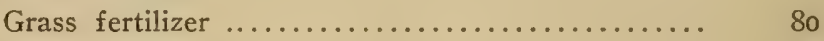

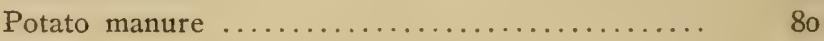

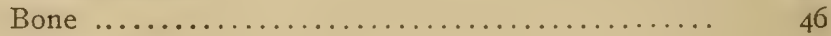

Tankage .............................. 50

Acid phosphate ..................... 40, $4 \mathrm{I}$

Chrysanthemum fertilizer ................ 98

Muriate of potash ................. 44, 45

Nitrate of soda .................... 23

Special mixture ................... 80, 84

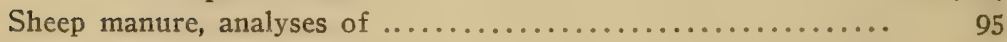

Shoddy waste, analyses of $\ldots \ldots \ldots \ldots \ldots \ldots \ldots \ldots \ldots . \ldots \ldots$

Shoemaker, M. L., \& Co. :-

"Swift-Sure" Superphosphate for general use .......... 80

potatoes ............. 80

truck; corn and onions .. 80

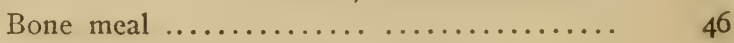

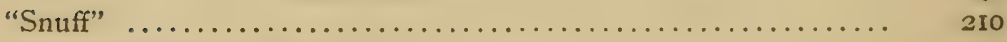

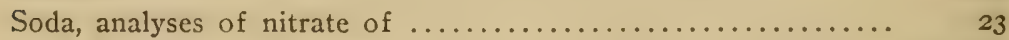

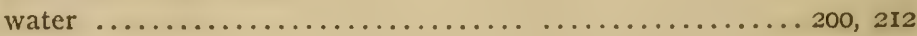

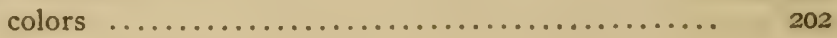


Page

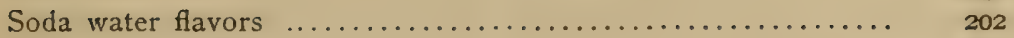

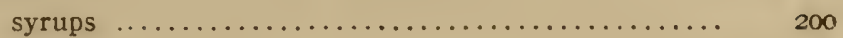

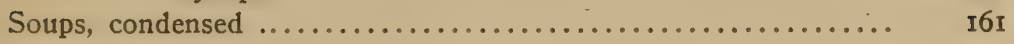

Sphecius speciosus ............................ 303

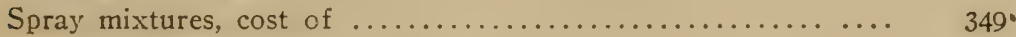

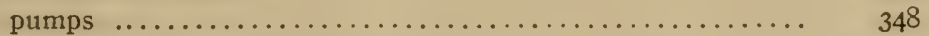

Sprayers, gasoline power ........................ 348

Sprays on apples, peaches, etc., tests of summer ........... 347

Steero Bouillon Cubes ............................ I62

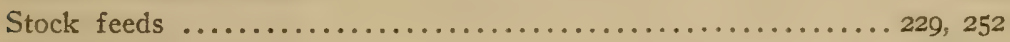

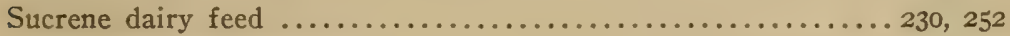

Sulphur and lead arsenate ....................... 367, 367, 369

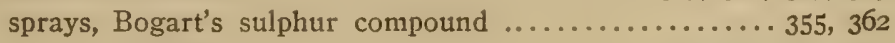

One-for-all ........................... 355, 362

Sulfocide ................... 355, 362, 369, 373

Swift's Lowell Fertilizer Co.:-

Swift's Lowell Animal brand ................... 80

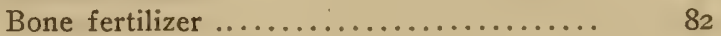

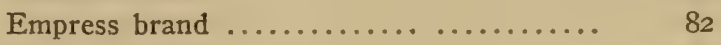

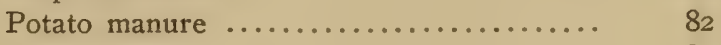

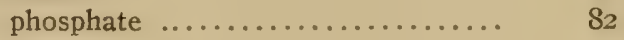

Perfect tobacco grower ................. So

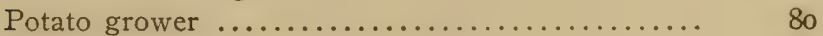

Special corn and vegetable manure ........... 80

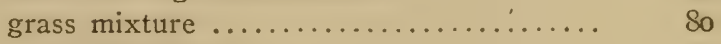

potato fertilizer ................. So

Superior fertilizer ................. 80

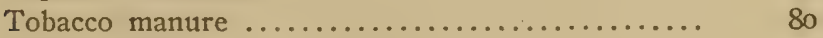

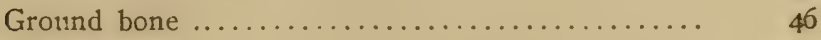

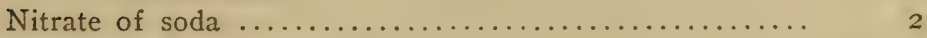

Tankage, analyses of slaughter house .............. 49, 50

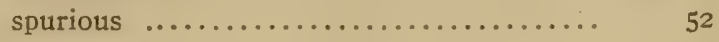

method of valuation $\ldots \ldots \ldots \ldots \ldots \ldots \ldots \ldots \ldots \ldots \ldots$

Tanner \& Wilcox:-

Tanner \& Wilcox' Reliable potato and garden phosphate .. 82

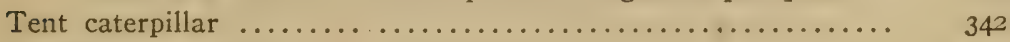

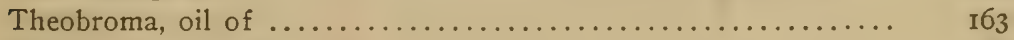

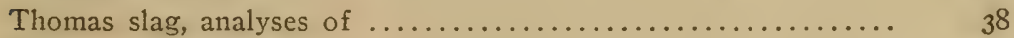

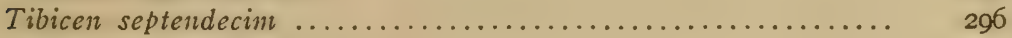

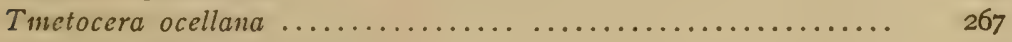

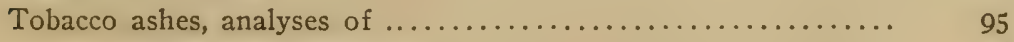

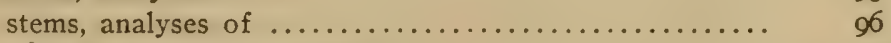

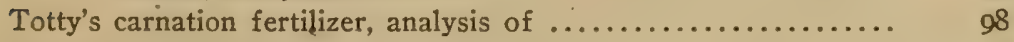

Trade values of fertilizer elements in I9II ................ 6

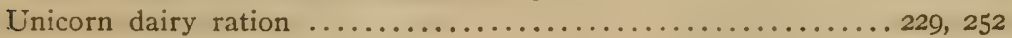

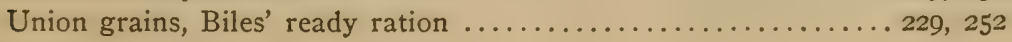


Valuation of bone and tankage, method of

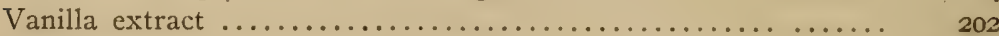

Vegetable potash, analysis of ...................... 46

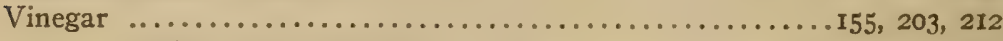

methods of analysis of $\ldots \ldots \ldots \ldots \ldots \ldots \ldots \ldots \ldots . . . . .157$

Weather conditions, effect on spraying ................ 363

Wells \& Downs:-

W. \& D. Special economical $6-8-5 \ldots \ldots \ldots \ldots \ldots \ldots \ldots . . . . . .62$

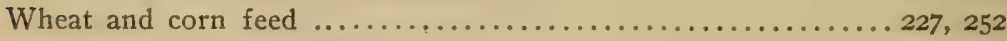

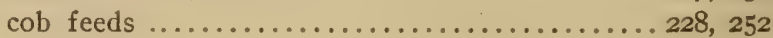

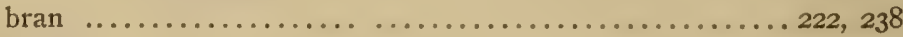

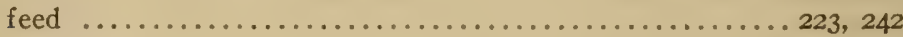

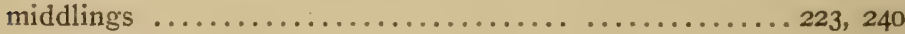

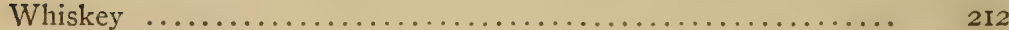

White pine weevil .............................. 268, 307

Wilcox Fertilizer Co.:-

Wilcox Complete bone superphosphate ............ 82

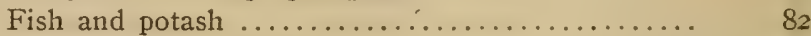

4-8-ro fertilizer ...................... 82

Grass fertilizer ................................... $8_{2}$

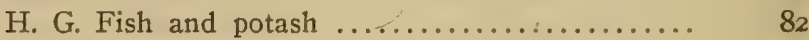

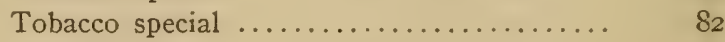

Potato fertilizer ..................... $8_{2}$

onion and vegetable phosphate $\ldots \ldots \ldots \ldots .82$

Special superphosphate .................. 82

Dry ground acidulated fish $\ldots \ldots \ldots \ldots \ldots \ldots \ldots$ 54, 55

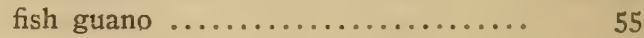

Ground steamed bone .................. 2

H. G. Tankage .................. 49, 50

Pure ground bone .................... 46

Acid phosphate ..................... 40, 4 I

Basic slag meal ........................ 38

H. G. Sulphate of potash ............... 43

Muriate of potash .................... 44

Nitrate of soda ...................... 23

Browning's formula .................. 84

Fielden's formula I $\ldots \ldots \ldots \ldots \ldots \ldots \ldots \ldots \ldots . \ldots \ldots$

$2 \ldots \ldots \ldots \ldots \ldots \ldots \ldots \ldots \ldots \ldots$

I, grain and potatoes ........ 84

2 , grass . . . . . . . $84 \ldots \ldots \ldots$

Wine of the extract of cod liver oil ................... I7I

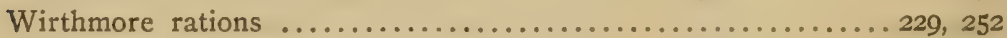

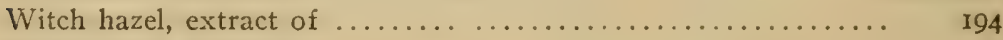

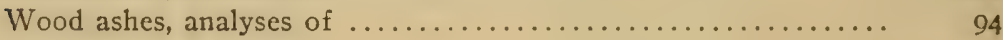


Woodruff, S. D., \& Sons:-

Woodruff's Home mixture

58,82

Worcestershire and other sauces

Zeusera asculi

Zinc, ortho-arsenite of 





\section{Itate of Connecticut}

\section{PUBLIC DOCUMENT No. 24}

\section{Thirty-fifth Annual Report}

OF

\section{The Connecticut Agricultural Experiment Station}

Being the annual report for the year ended October 31

\section{1}

PRINTED BY ORDER OF THE LEGISLATURE 


\section{PUBLICATION APPROVED BY THe BoARd oF Control.}




\title{
CONNECTICUT AGRTCULPURAL EXPERIMENT STATTON.
}

\author{
OEFIOERS AIND STAFF'-
}

September 30, I9I I

BOARD OF CONTROL.

His Excellency, Simeon E. Baldwin, ex officio, President.

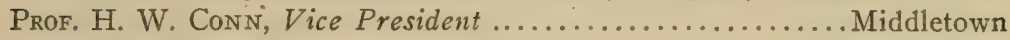

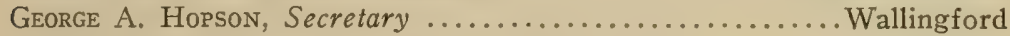

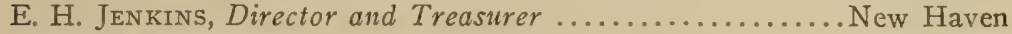

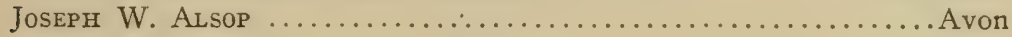

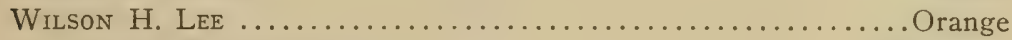
Frant H. Stadtmuellet $\ldots \ldots \ldots \ldots \ldots \ldots \ldots \ldots \ldots \ldots \ldots \ldots \ldots \ldots \ldots$ Elmwood

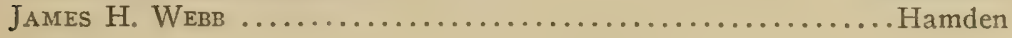

\section{AdMINistration.}

Chemistry.

Analytical Laboratory. John Phillips Street, M.S., Chemist in Charge.

Proteid Research.

BOtANx.

ENTOMOLOGY.

FORESTRY.

Plant Breeding.

Buildings and Grounds. E. Monroe Bailey, Ph.D., C. B. Morrison, B.S., R. B. RoE, A.B., C. E. Shepard, Assistants. Hugo LANGE, Laboratory Helper.

V. L. Снurchilı, Sampling Agent.

\section{STATION STAFF.}

E. H. Jenkins, PH.D., Director and Treasurer. Miss V. E. Cole, Librarian and Stenographer. Miss L. M. BrautLeCHT, Bookkeeper and Stenographer.

T. B. OsBorne, PH.D., Chemist in Charge. Miss E. L. Ferry, A.B., Assistant. Miss Luva Francis, Stenographer.

G. P. Clinton, S.D., Botanist.

E. M. Stoddard, B.S., Assistant. Miss M. H. JAGger, Seed Analyst.

Miss E. B. Whittlesey, Herbarium Assistant.

W. E. Britton, Ph.D., Entomologist; also State Entomologist.

B. H. Walden, B.Agr., D. J. Caffreey, B.S., A. B. Champlain, Assistants.

Miss E. B. Whittlesey, Stenographer.

Samuer N. Spring, M.F., Forester; also State Forester and State Forest Fire Warden. W. O. Filley, Assistant; also Asst. State Forester. Miss E. L. Avery, Stenographer.

H. K. Hayes, B.S., Plant Breeder.

C. D. HubBel, Assistant.

William Veitch, In Charge. 



\section{TABLE OF CONTENTS.}

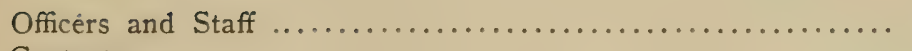

PAGE

Contents

iii

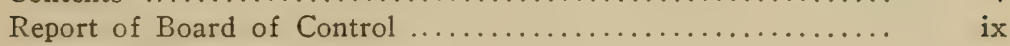

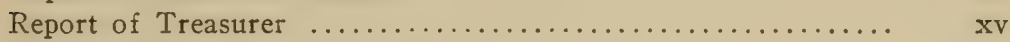

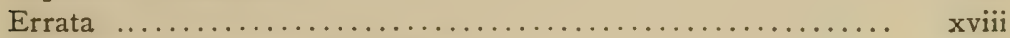

Report on Commercial Fertilizers $\ldots \ldots \ldots \ldots \ldots \ldots \ldots \ldots \ldots$ I

The Fertilizer Law and its Observance ............... I

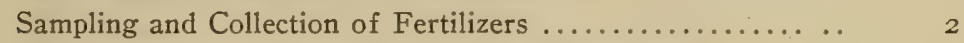

Explanations concerning Analyses and Valuations ........ 3

The Solubility of Organic Nitrogen ................ 8

A Modified Method to determine Nitrogen Solubility ........ 9

Solubility of the Organic Nitrogen of Raw Materials ...... I2

Pot Experiments on Nitrogen Availability .............. I4

Analyses of Raw Materials chiefly valuable for Nitrogen ... 23

Raw Materials chiefly valuable for Phosphoric Acid .... 38

Raw Materials chiefly valuable for Potash .......... 42

Raw Materials containing Nitrogen and Phosphoric Acid 47

Mixtures of Phosphates with Potash Salts ........... 54

Nitrogenous Superphosphates ................ 56

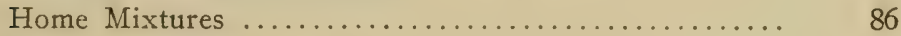

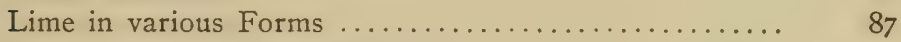

Cotton Hull and ther Ashes ................ 92

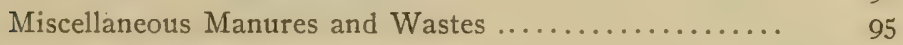

Report on Food Products ........................ IOI

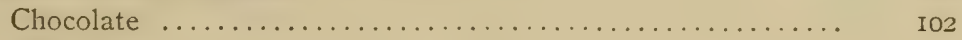

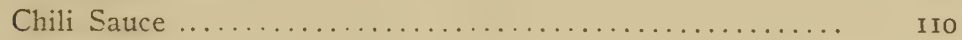

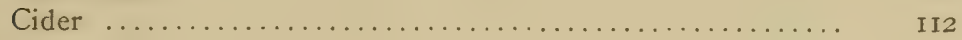

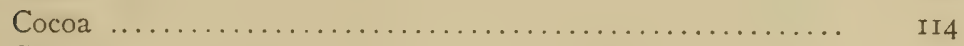

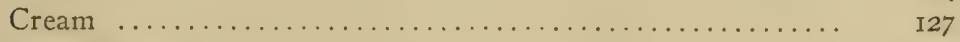

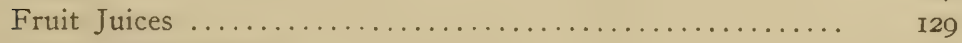

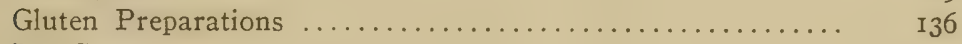

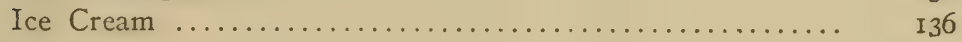

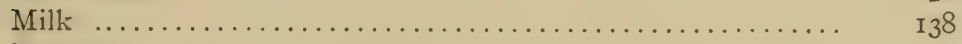

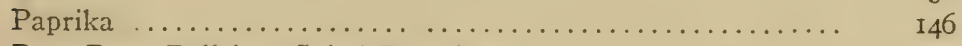

Root Beer, Relishes, Salad Dressing ............... 150

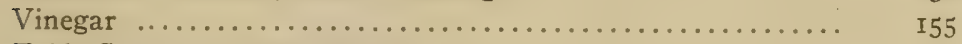

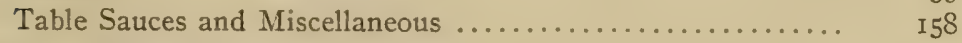

Report on Drugs .......................... I62

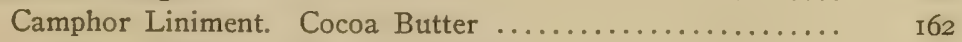

Cod Liver Oil and other Emulsions ................ 163

Wine of the Extract of Cod Liver Oil .................... I7

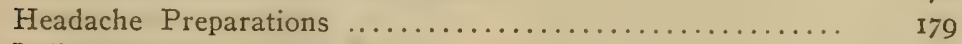

Iodine Tincture. Lime Water .................... I80 
Report on Drugs, cont'd.

PAGE

Spirit of Nitrous Ether ....................... I8I

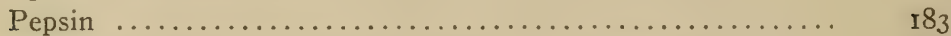

Quinine Pills and Compound Extract of Sarsaparilla ...... I88

Extract of Witch Hazel ...................... 194

Food and Drug Products examined for the Dairy Commis-

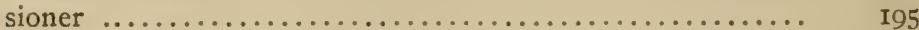

Miscellaneous Foods and Drugs ................. 210

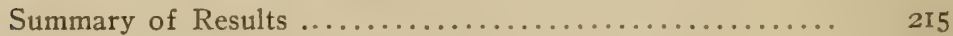

Report on Commercial Feeding Stuffs ................. 2 I9

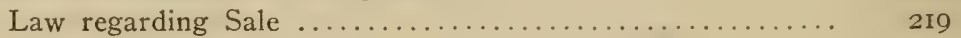

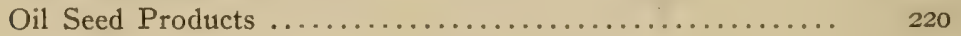

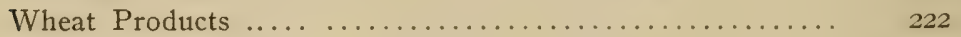

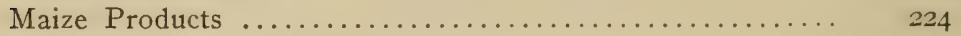

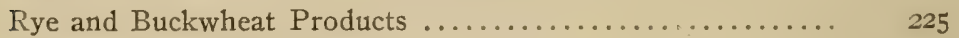

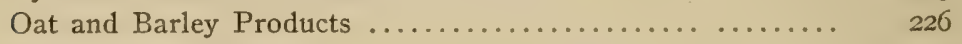

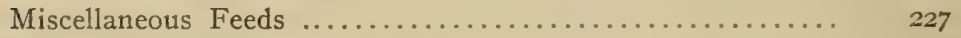

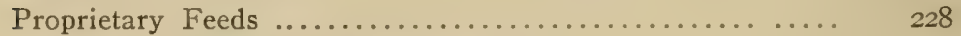

Digestibility of Feeds by Ruminants ................. 233

Average Composition Digestibility and Selling Price of Feeds 233

Notes regarding the Yield of Alfalfa ................ 237

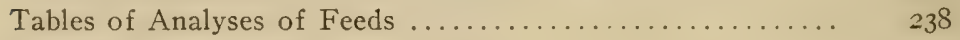

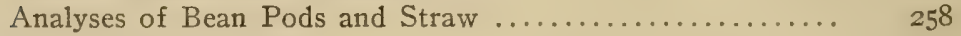

Report of the Entomologist ...................... 259

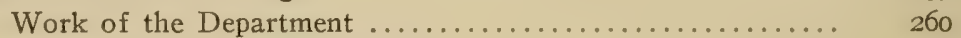

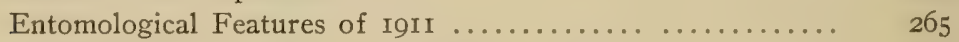

Inspection of Nurseries and Imported Stock ........... 269

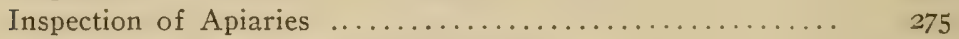

Progress in Controlling the Gypsy Moth .............. 277

Checking the Spread of the Brown-Tail Moth .......... 28I

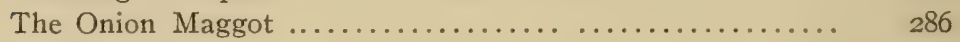

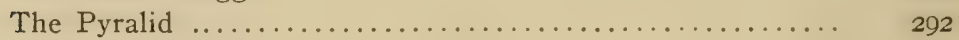

The Seventeen-Year Locust ................... 296

The Maple Leaf-Stem Borer .................... 305

Preliminary Tests to Prevent Damage by the White Pine

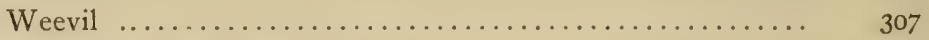

The Cherry Tent Maker ....................... 309

The Poplar Mocha-Stone Moth ................... 310

The Colorado Potato Beetle .................... 3II

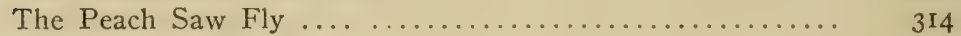

How to get rid of Ants .............................. 3 I6

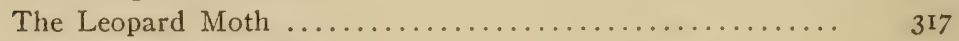

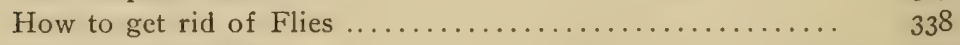

Brief Insect Notes ......................... 339

Report on Tests of Summer Sprays on Apples, Quinces, etc. .... 347

Types of Sprays used ........................ 349

General Results with Apples ................... 358 
Report on Tests of Summer Sprays on Apples, etc., cont'd.

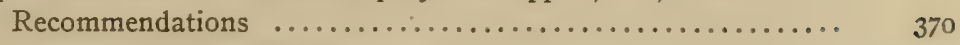

General Results with Peaches .................. 371

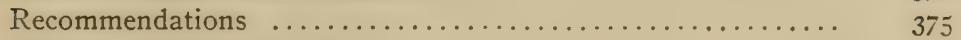

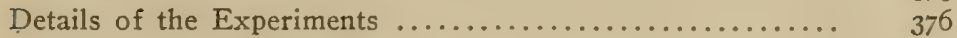

Experiments with Pears and Quinces ............... 396

Cherries and Plums .............. 398

Currants .................... 405

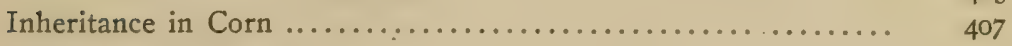

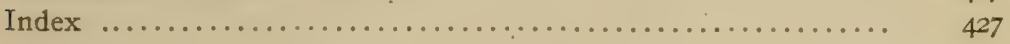





\section{REPORT OF THE BOARD OF CONTROL}

$\mathrm{OF}$

\section{THE CONNECTICUT AGRICULTURAL EXPERIMENT STATION.}

To His Excellency, Simeon E. Baldruin, Governor of Connecticut:

The Board of Control of The Connecticut Agricultural Experiment Station herewith respectfully submits its report for the year ending November I, I9II.

The following minute regarding William Henry Brewer, for many years a member of this Board, was adopted by the Board at its meeting on December 22, I910:

Professor William H. Brewer, Ph.D., LL.D., a member of this Board for thirty-three years, died at his home in New Haven on November 2, 1910. His manifold work as geographer, botanist, sanitarian and teacher needs no recital here.

As one of the pioneers in bringing agricultural science to the knowledge of farmers and teaching its value for practical ends, his name will be held in grateful remembrance by the farmers of this State.

Born and brought up on a farm and having personal experience of farm work, he began his studies in Yale College with the object of fitting himself for the life of a farmer. Drawn by his tastes for natural science and eagerness to follow up the studies he had begun, he finished the regular course and graduated at the Sheffield Scientific School in 1852. After studying abroad at Paris, Heidelberg and Munich, serving as a college professor at Washington College, Pennsylvania, and the University of California, and working for four years on the survey of California, he returned to the Scientific School of Yale as professor of agriculture in 1864 , and held that position until his retirement as professor emeritus in 1903.

$\mathrm{He}$ was professor of agriculture not only within college halls but everywhere in the State. His intimate acquaintance with practical farming, his scientific attainments, the wide range of his knowledge and of his interests, together with his broad common sense, made him a welcome and effective speaker at farmers' gatherings everywhere.

He was earnest and influential in establishing this Agricultural Experiment Station and he served it wisely and effectively until he died. His last public act was to attend a meeting of this Board only a few days before his death. 
Probably no one in the University during the last fifty years was called on so often and so variously for public service outside of the institution as Professor Brewer, and in all of it he was faithful, tactful and efficient.

The Members of THIS BoARd wish here to record their sense of personal loss, their appreciation of his rare qualities as a friend and adviser, and of his long and effective labors for the advance of agriculture.

It Is ORDERED that this minute be entered on the records of this Station and that a copy be sent to Professor Brewer's family.

A detailed account of the work of each of the several departments of the Station will appear in the reports of individual members of the staff, which are now in preparation.

We therefore only call attention to certain facts relating to the Station welfare which will not be discussed in those publications.

The General Assembly, at the January session of I9I I, appropriated $\$ 6,500$ to this Station to cover the loss caused by the fire of January ro, I9ro.

Chapter 192 of the Public Acts of I9II provides for the printing of an annual report of this Station, which had been the practice for thirty years, in place of the biennial report required by statute during the last four years.

Chapter I34 of the Public Acts of I9II requires that the net contents of all packages of food products shall be plainly marked on the outside in terms of weight, measure, or numerical count, and that the director of this Station, with the Dairy and Food Commissioner, shall establish rules and regulations as to the reasonable variations and allowances which shall be permitted. No penalty is to be enforced prior to eighteen months from the passage of this Act.

Chapter 274 forbids adulteration of turpentine or spirits of turpentine and makes it the duty of the Dairy and Food Commissioner and the director of this Station "acting jointly" to enforce this law. To the Commissioner alone is given the right of inspection. This statute, unlike any other relating to adulteration, makes the Station jointly responsible for enforcing the law. This is a departure from previous policy. Hitherto the Station's responsibility has been limited to testing suspected 
articles, reporting its findings to the Commissioner, and giving expert evidence in court when required.

There have been no changes of importance in the Station staff except that Mr. Walter O. Filley has been appointed assistant state forester, an office created by the last General Assembly, and Mr. A. B. Champlain, an assistant in the entomological department, resigned October I, I9II, to accept a position in the State Laboratory of Economic Zoology of Pennsylvania.

During the fall, an exhibit illustrative of the several departments of the Station work was made at six agricultural fairs and also at the Station grounds in New Haven, the last especially for the teachers of the State who were attending a convention at the time in New Haven. Members of the Station staff were in constant attendance at all these exhibits to explain them and to answer questions. This involved the almost complete suspension of our regular laboratory work for nearly six weeks, which was a serious interruption. The large attendance, however, and the interest shown in the Station work as illustrated by the exhibits convince us that this is one of the most effective ways of diffusing information regarding our work among the farmers of the State.

The Station has bought a farm of twenty acres at Mount Carmel, not far from the city, including a small house for the caretaker, has set out an orchard of apples and peaches for experiment, has begun an experiment on the effect both on the crop and on the soil of fertilizers and manures, and another, on the handling of an old and neglected orchard.

At the Centerville field, which will be given up in the spring of I9I2, we have over 800,000 white pine seedlings, three years old, which are being sold at cost to citizens of this State for forest planting.

On this field also corn and tobacco breeding work, as well as other experiments, has been carried on during the present year. Hereafter this work will be concentrated on our own fields at Mount Carmel.

The Station receives one-half of the so-called Adams Fund which, by Act of Congress and the rulings of the Secretary of Agriculture, must be used wholly for scientific research, preferably in a small number of "projects" approved by the Office of 
Experiment Stations. The Station's share of this fund is devoted to two projects: a study of the laws of inheritance in maize and tobacco, and a study of the composition, structure and relative nutritive value of the vegetable proteins. This last project, which has engaged Dr. Osborne's time for many years, now receives very substantial aid from the Carnegie Institution of Washington.

Bulletin 167 , Inheritance in Maize, gives an account of five years' study of the subject, and Bulletin I68 is a discussion of the facts discovered which may be of immediate practical account. The results of work on the other project are published in physiological and chemical journals and in monographs of the Carnegie Institution and are not further noticed in this report.

During the year there have been issued parts of a biennial report aggregating 512 pages with $2 x$ plates in editions of 10,000 copies, and three bulletins aggregating I74 pages with 30 plates. Nine thousand five hundred copies of one of these were printed, of another three thousand, but of the third, which was so technical as to be of value chiefly to investigators, only one thousand were printed. The spray calendar has also been revised to include the results of recent work and is mailed as requested.

Fifty-one addresses have been given by members of the staff at farm institutes, field meetings, granges and other gatherings of farmers, and the Station correspondence has involved the writing of more than 9400 letters and manuscript reports.

The following summary shows in brief the departments of the Station work and the special directions it has taken:

\section{Entomological Department.}

Inspection of all the nurseries in the State and of imported nursery stock to prevent distribution of insect and fungous pests; inspection of apiaries on request or complaint; gypsy moth control work at Stonington and Wallingford; search for the browntail moth and destruction of its winter nests in northeastern Connecticut; studies of life-histories of certain insects, preparation of exhibits; and publications in various journals on entomological subjects; also coöperation with the botanical department in the study of summer sprays, which is described below. 
Botanical Department.

The preparation of artificial cultures of many fungi, most of them of economic importance, for purposes of investigation; studies on the oöspore production of the potato blight in artificial cultures and the relation of media to the stages and character of growth of fungi in artificial cultures; publication of . papers on botanical subjects; studies in the field of the chestnut blight, calico of tobacco and peach yellows; tests of Millar's Cream muskmelon; and spraying experiments with melons, cucumbers and potatoes.

In coöperation with the Entomological Department, very extensive and careful tests have been made to determine the comparative value of the various summer sprays on apples, peaches, pears, plums, cherries and some other fruits. The tests were made in thirteen orchards and involved the individual examination of 93,000 apples and about 25,000 peaches.

\section{Seed Testing.}

Tests of purity and vitality of field and garden seeds and identification of weed seeds for farmers and dealers; and studies of methods of seed testing.

\section{Forestry Department.}

The care of the three Station nurseries, containing about I, IOO,000 young trees for forest planting; care of the Station forest plantations, including forest planting, cleaning out worthless species and liberation cutting; a test of basket willows; a demonstration planting of red and white pine at Putnam Memorial Camp Ground; inspection of State forests and destruction of pine weevil; making fire lines and improvement thinning at the Portland forest; the careful gathering of statistics of the 828 forest fires of the year which caused damage amounting to more than $\$ 235,000$. New and desirable legislation has been secured regarding forest fires and the work of the forest fire wardens further systematized. Examinations of woodland for private owners have been made and advice given regarding planting, thinning and cutting. In coöperation with the U. S. Forest Service a study is being made of the woodworking industries of 
the State, to get if possible a better utilization of home-grown woods and give the woodland owner information as to markets for his product.

\section{Chemical Department.}

Analyses and published reports of all commercial fertilizers sold in the State, of commercial feeds, and of human food products and drugs; examinations of foods and drugs for the Dairy and Food Commissioner, and expert evidence in court as required; study of methods of analysis; and analytical work required in connection with field experiments.

\section{Plant Breeding.}

(Supported by Adams Fund)

Studies of inheritance of characters in corn and tobacco and of the yields of first year corn hybrids.

\section{PRotein RESEARCH.}

(Supported by Adams Fund)

Studies of the composition, structure and relative nutritive values of the vegetable proteins.

The above list of the important parts of the Station work illustrates how it covers the whole State and affects the interests of all its citizens, whether farmers or not.

Each session of the General Assembly adds to the State's requirements of the Station, the calls made by farmers and others for the help which it is the object of a Station to give, and the natural and inevitable expansion of its work have made the Station's income insufficient for its needs. The strictest economy will be necessary for the next year, and without a larger income than it now has the work which it is doing must thereafter be lessened and its working force cut down. For the general expenses of the Station no increase of appropriation has been made by the State since 1895 .

All of which is respecctfully submitted.

George A. Hopson, Secretary. 


\section{REPORT OF THE TREASURER, 1911}

E. H. Jenkins, in account with The Connecticut Agricultural ExperiMent Station for the fiscal year ending September 30, I9Ir.

RECEIPTS.

Balance on hand, October I, I9Io (State Agricultural Appropriation)

State Appropriation, Agriculture $\ldots \ldots \ldots \ldots \ldots \ldots \$ 10,000.00$

State Appropriation, Food .................. 2,500.00

State Appropriation, Insect Pest .............. 3,000.00

State Appropriation, Gypsy Moth ............. 4,000.00

United States Appropriation, Hatch ........... 7,500.00

United States Appropriation, Adams ........... 7,500.00

Analysis Fees ......................... 12,500.00

Sale of Farm Products ................... 80.79

Miscellaneous Receipts ................... 339.8I

From the Lockwood Estate .................. 16,611.23

Total $\$ 1,655.26$

\section{DisBuRSEMENTS.}

E. H. Jenkins, director, salary .............. $\$ 2,800.00$

E. H. Jenkins, treasurer, " $\ldots \ldots \ldots \ldots \ldots \ldots .400 .00$

W. H. Brewer, salary ...................... 8.34

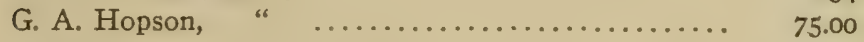

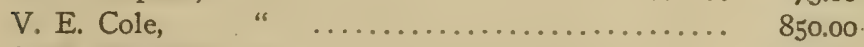

L. M. Brautlecht, " $\ldots \ldots \ldots \ldots \ldots \ldots \ldots \ldots \ldots .750 .00$

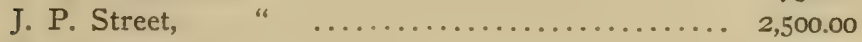

T. B. Obsorne, " $\quad \ldots \ldots \ldots \ldots \ldots \ldots \ldots \ldots \ldots$. $2,400.00$

E. M. Bailey, " $\quad \ldots \ldots \ldots \ldots \ldots \ldots \ldots \ldots \ldots \ldots \ldots \ldots \ldots$ I,550.00

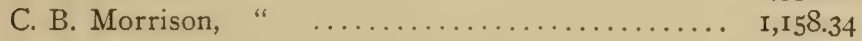

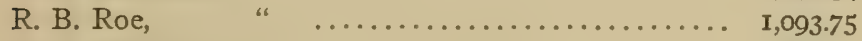

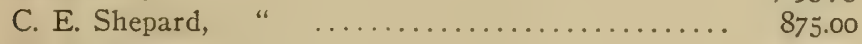

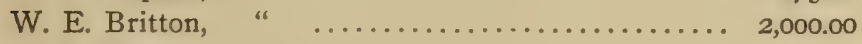

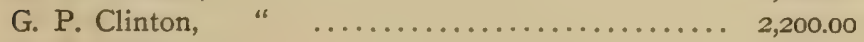

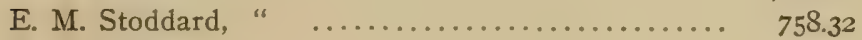

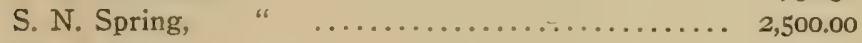

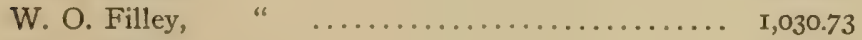

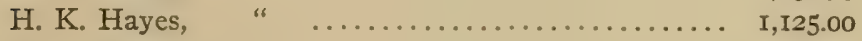

E. L. Ferry, " $\quad$ " . . . . . . . . .

H. Lange, " $\quad$ "..................... 925.00

V. L. Churchill, " $\ldots \ldots \ldots \ldots \ldots \ldots \ldots \ldots \ldots . . . \ldots 25.00$

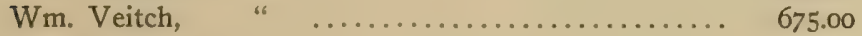

Luva Francis, " .................... 350.00 
E. L. Avery, salary

H. W. Kiley (Labor)

$\$ 390.00$

Wm. Pokrob

728.00

C. D. Hubbell

728.00

728.00

Geo. Graham

724.00

M. H. Jagger

499.00

I 80.00

475.00

I73.00

L. Nolan

$3,842.79$

Labor

I, I 37.82

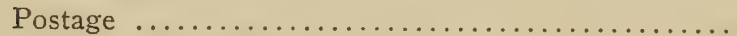

$357.7 \mathrm{I}$

709.31

161.69

296.30

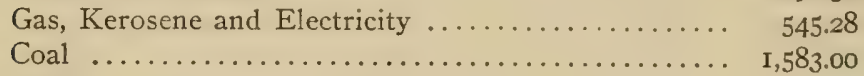

Water

I34.44

Chemicals and Laboratory Supplies ........... 897.82

Agricultural and Horticultural Supplies ......... 2,567.05

Miscellaneous Supplies .................. $\quad 575.76$

Fertilizers ......................... 480.51

Feeding Stuffs ........................ I88.98

Library and Periodicals ................. 843.58

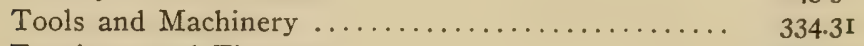

Furniture and Fixtures ................. $\quad 294.00$

Scientific Apparatus ....................... ${ }_{21} 8.02$

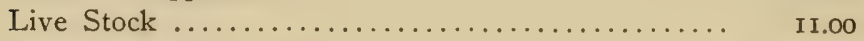

Traveling by the Board $\ldots \ldots \ldots \ldots \ldots \ldots \ldots \ldots$ II8.II

Traveling by the Staff ................ I,295.9I

Traveling in connection with Adams Fund Investi-

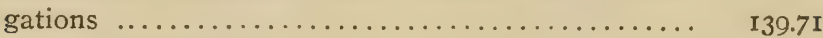

Fertilizer Sampling $\ldots \ldots \ldots \ldots \ldots \ldots \ldots \ldots \ldots \ldots$ 231.60

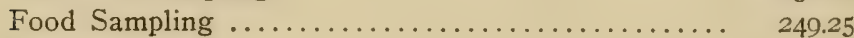

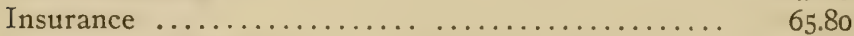

Insect Pest Appropriation to State Entomologist ... 3,000.00

Contingent ............................ 572.51

Lockwood Expenses .................... 400.00

Gypsy Moth Appropriation to State Entomologist ... 4,000.00

New Buildings ...................... 396.90

Betterments ....................

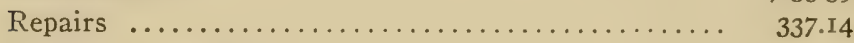

Rental of Land ....................... 105.00

Purchase of Land ................. $6,000.00$

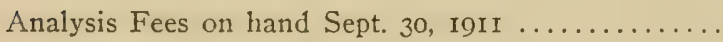


New Haven, Conn., Nov. I4th, Igir.

THIS Is To CERTIFy that we have examined the accounts of E. $H$. Jenkins, Treasurer of The Connecticut Agricultural Experiment Station, for the year ending Sept. 30th, I9II, have compared the same with the vouchers therefor and found them correct.

\author{
William P. Bailey, \\ EDWARD S. ROBERTS, \\ Auditors of Public Accounts.
}

E. H. Jenkins, in account with The Connecticut Agricultural ExperiMent Statron for the fiscal year ending Sept. 30, I9II.

Receipts and Disbursements in connection with the rebuilding of the laboratory building destroyed by fire January 10, I910.

RECEIPTS.

Balance on hand Oct. I, Igro............... \$I

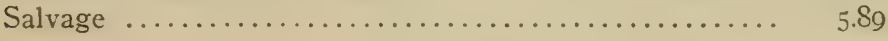

\title{
Disbursements.
}

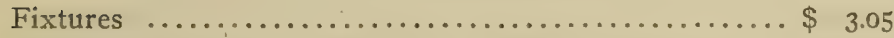

Apparatus, Laboratory Supplies and Miscellaneous ..... 22.97

Part of interest on loan of $\$ 6,000 \ldots \ldots \ldots \ldots \ldots \ldots . . . \ldots \ldots$

This Certifies that we have examined the account which relates to loss by fire and rebuilding new building, have compared the same with the vouchers therefor and find them correct, the said account being closed and balanced by the expenditure of all moneys in the account, same being evidenced by vouchers on file and bank book.

William P. Bailey,

Edward S. Roberts, Auditors of Public Accounts. 
XViii CONNECTICUT EXPERIMENT STATION REPORT, IOII.

\section{ERRATA.}

Page 124, Stollwerck's Milk Cocoa is stated to contain glucose. This statement is an error. There is no evidence of the presence of glucose or other adulterant in this brand.

Page 200, nineteenth line from top, for henzoic read benzoic.

Page 227, “The Biles' product" mentioned under Dried Distillers' Grains refers only to rye grains, the analysis of which is given on page 250 , and not at all to the other and higher grade brands of distillers' grains sold by the Biles Company.

Page 229. The manufacturers state that Husted's Steam Cooked Feed contains only whole and cracked corn and whole oats but no wheat. 







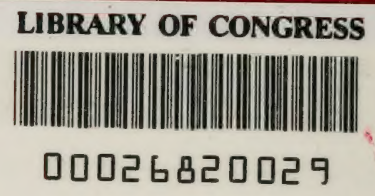

